\title{
The Long-Baseline Neutrino Facility
}

David Montanari

ECD \& IWC-HTS 2017

Sep 13-15, 2017 


\section{Outline}

- Intro.

- Scope and Strategy.

- Modes of Operations.

- Requirements.

- Cryostat.

- Cryogenic Systems.

- LAr Procurement.

- Schedule.

- Summary. 


\section{Intro: The LBNF/DUNE Project}

- The Long-Baseline Neutrino Facility is the infrastructure necessary to send a powerful beam of neutrinos 800 miles through the earth, and measure them deep underground at South Dakota's Sanford Underground Research Facility (SURF). LBNF supports DUNE.

- The Deep Underground Neutrino Experiment will be a game-changing experiment for neutrino science, potentially transforming our understanding of why the universe exists as it does.

- The LBNF/DUNE project will be the first internationally conceived, constructed, and operated mega-science project hosted by the Department of Energy in the United States.

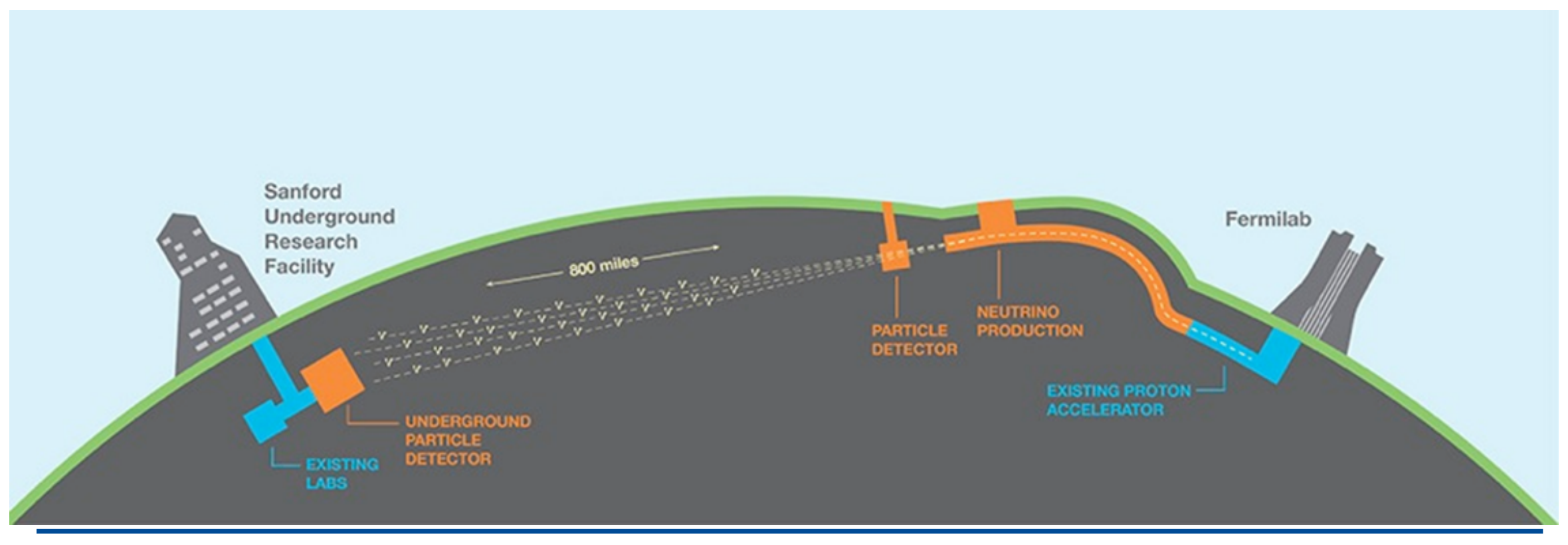




\section{The DUNE Collaboration}

Today:

$60 \%$ non-US

1011 collaborators from 164 institutions in 30 nations!!

- Armenia, Brazil, Bulgaria, Canada, CERN, Chile, China, Colombia, Czech Republic, Finland, France, Greece, India, Iran, Italy, Japan, Madagascar, Mexico, Netherlands, Peru, Poland, Romania, Russia, South Korea, Spain, Sweden, Switzerland, Turkey, UK, Ukraine, USA

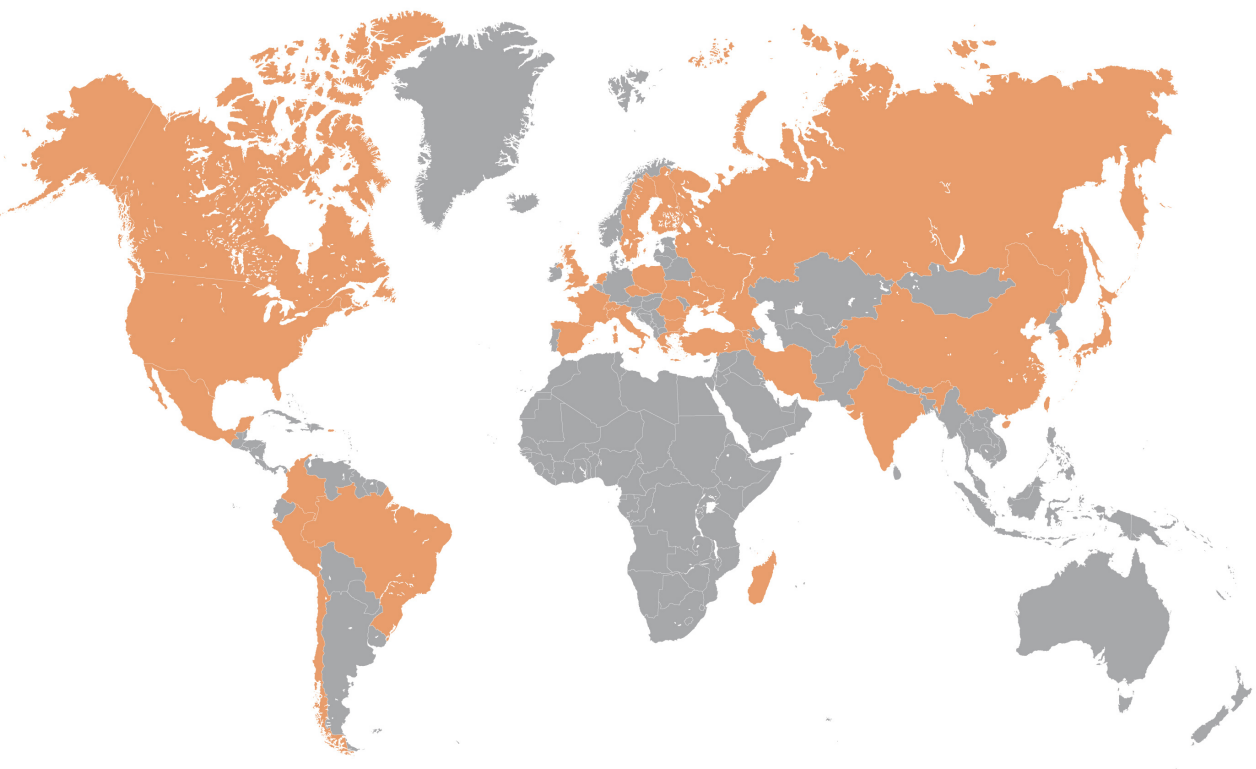

\section{DUNE is an International Experiment.}

\section{LBNF is an International Participation with Fermilab as the host Laboratory.}




\section{Ambitious, Far-Reaching Science Goals}
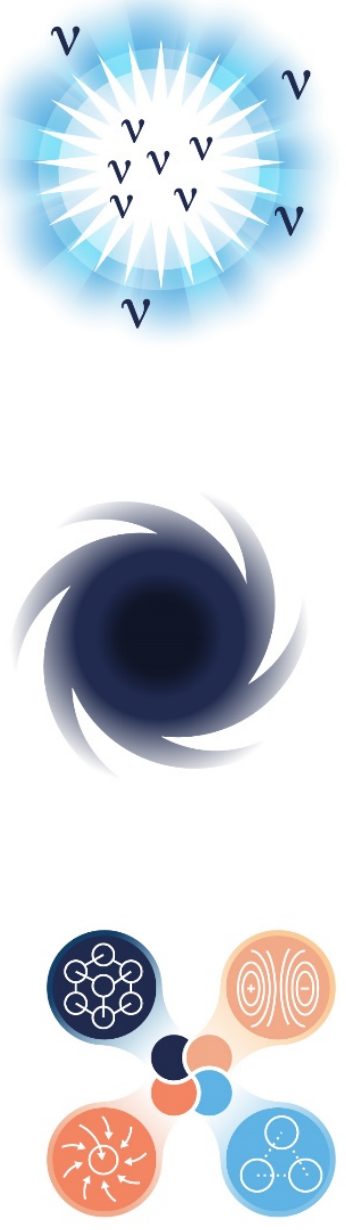

- Origin of matter Discover what happened after the big bang: Are neutrinos the reason the universe is made of matter?

- Black hole formation Use neutrinos to look into the cosmos and watch the formation of neutron stars and black holes in real time.

- Unification of forces Move closer to realizing Einstein's dream of a unified theory of matter and energy. 


\section{Neutrinos are Exciting Science!}

\section{The Nobel Prize in Physics}

\section{2}

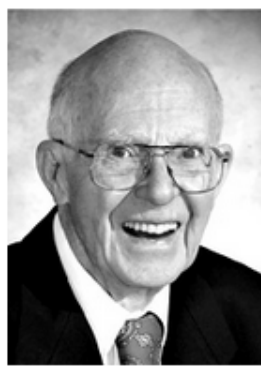

Raymond Davis Jr. Prize share: $1 / 4$

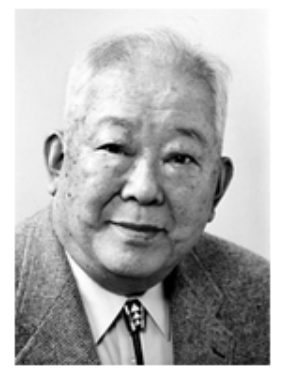

Masatoshi Koshiba Prize share: $1 / 4$

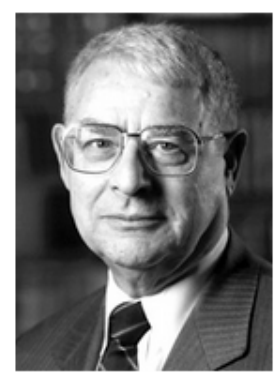

Riccardo Giacconi Prize share: $1 / 2$

\section{The Nobel Prize in Physics}

2015

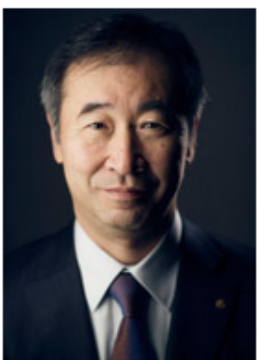

Photo: A. Mahmoud Takaaki Kajita Prize share: $1 / 2$

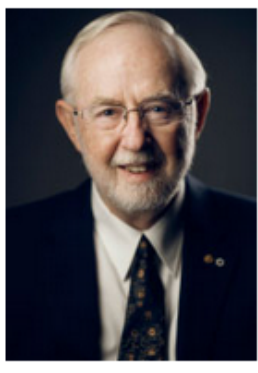

Photo: A. Mahmoud Arthur B. McDonald Prize share: $1 / 2$

The Nobel Prize in Physics 2002 was divided, one half jointly to Raymond Davis Jr. and Masatoshi Koshiba "for pioneering

The Nobel Prize in Physics 2015 was awarded jointly to Takaaki Kajita and Arthur B. McDonald "for the discovery of neutrino oscillations, which shows that neutrinos have mass" pioneering contributions to astrophysics, which have led to the discovery of cosmic X-ray sources".

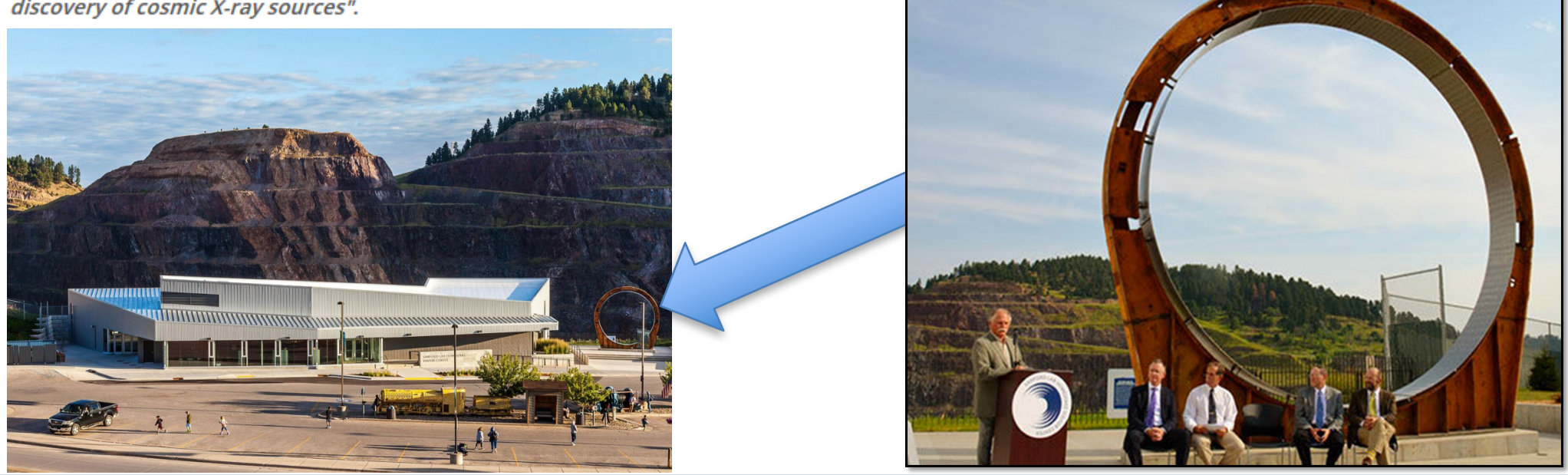




\section{LBNF}

- LBNF includes the Beam Line, the Conventional Facilities and the Cryostat and Cryogenics Infrastructure and has Facilities at two Locations:

- Near Site (at Fermilab, in Batavia, IL) where the Neutrino beam is generated in the accelerator complex, analyzed in the Near Detector and sent through the earth to the target at the Far Site.

- Far Side (at SURF, in Lead, SD) where an out-of-service gold mine (max original depth 8,000 ft) has been repurposed as a research facility. The $4,850 \mathrm{ft}$ Level is already in use for other scientific experiments and will be expanded for the LBNF/DUNE project. Here the Neutrino beam coming from Fermilab reaches its target: the Far Detector, composed of four tanks filled with LAr and Time Projection Chambers (TPCs) to measure the properties of Neutrinos among other things. Detection, Tracking \& Studying.

\section{Far Site (Lead, SD) \\ Near Site (Fermilab, Batavia, IL)}

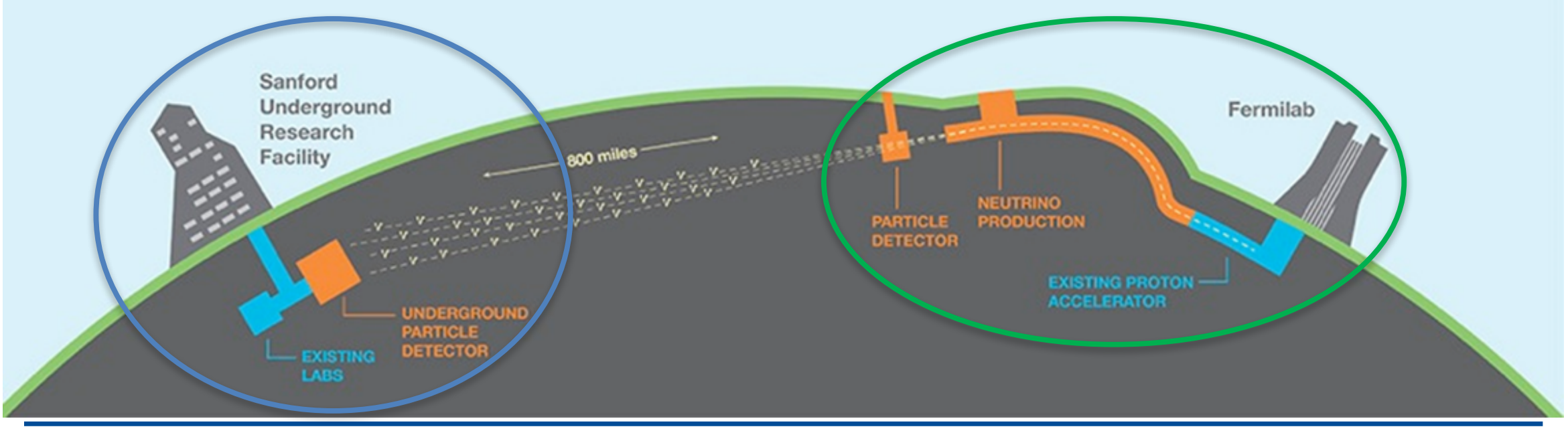




\section{Detector/Infrastructure Prototyping}

\section{ICARUS}

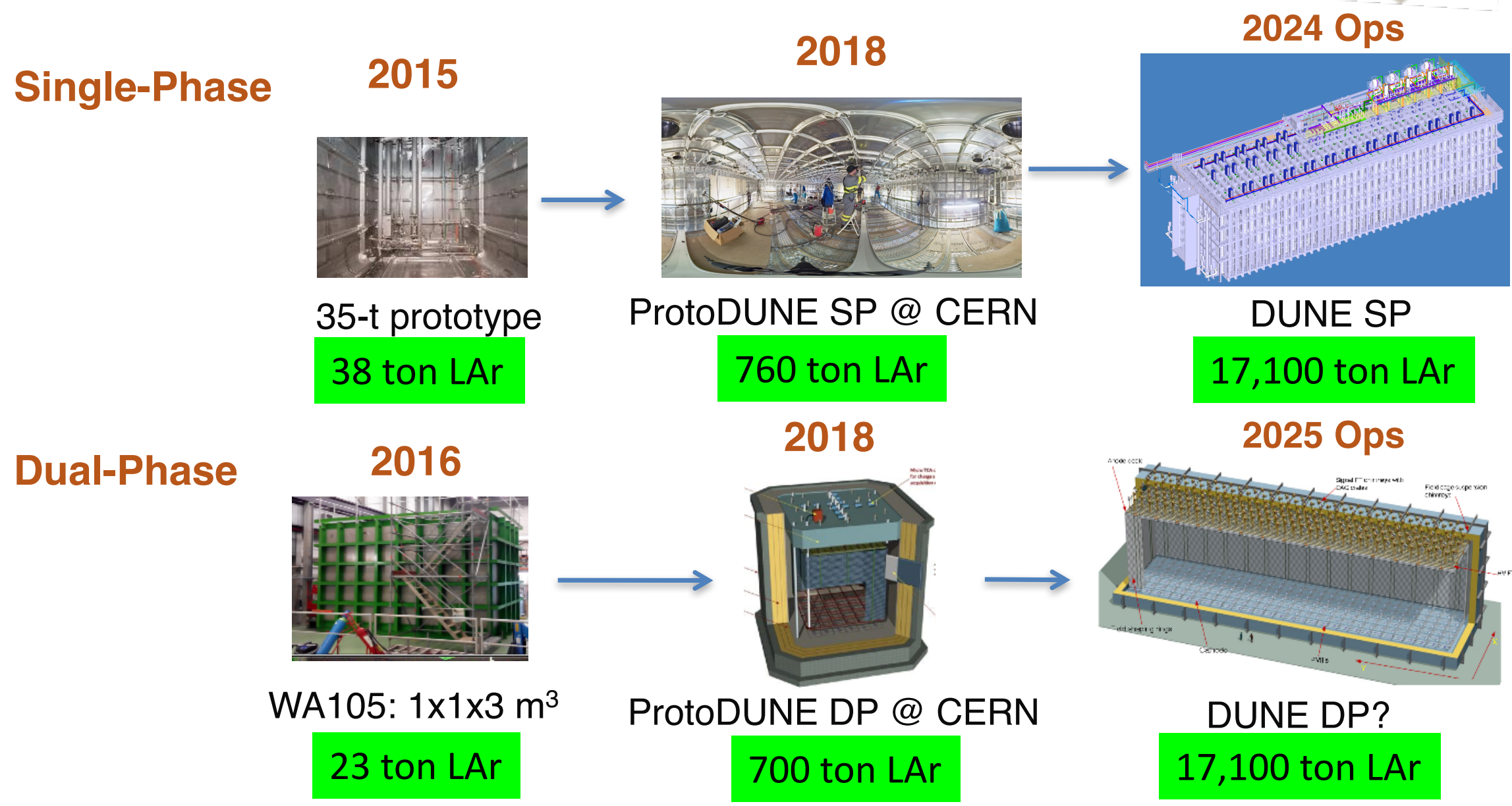




\section{SURF: Sanford Underground Research Laboratory (Lead, SD)}

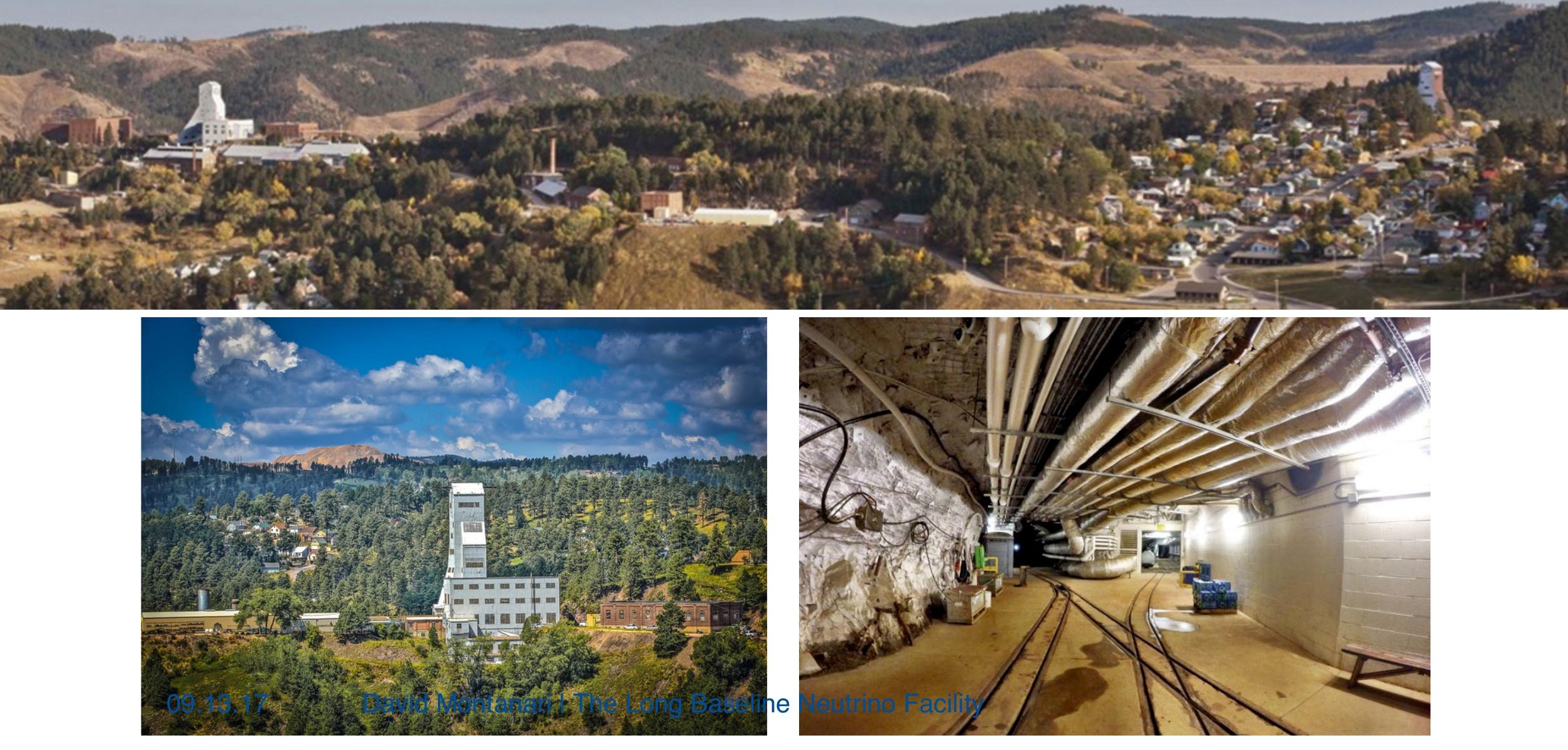




\section{Access Conditions at SURF}
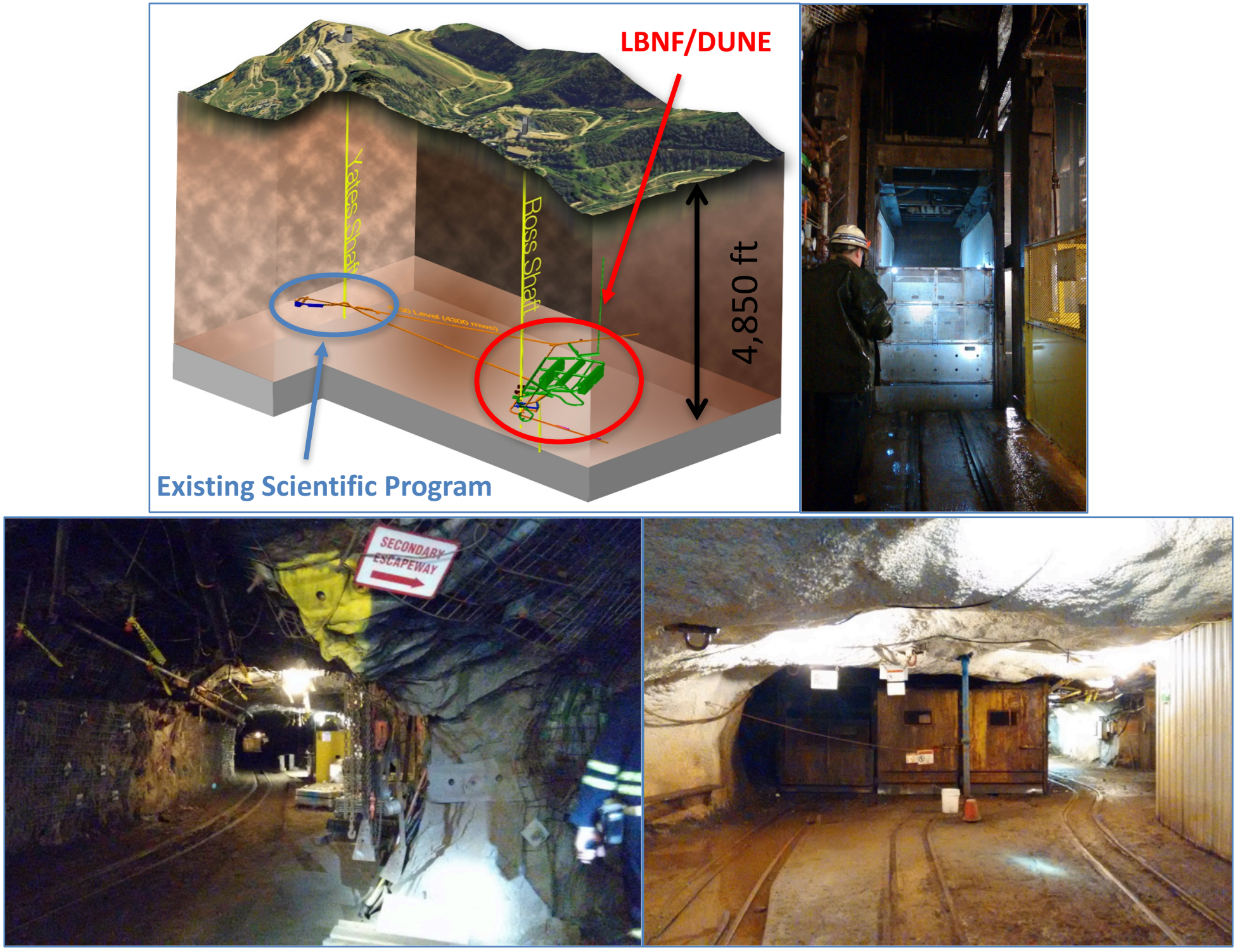


\section{Cryogenics Infrastructure Scope and Strategy}

- Cryogenics Infrastructure to support four 17-kton LAr mass detectors ( 70 kton total).

- International approach with CERN and Fermilab current main players, but there are very interesting opportunities for others to contribute.

- Cryostat includes membrane cryostat and steel support structure, the crane bridges in caverns for cryostat and support structure installation, mezzanines and their supports.

- Predominantly non-DOE. CERN responsible for $1^{\text {st }}$ Cryostat.

- Responsible parties for remaining cryostats are to be identified.

- Cryogenic Systems includes design, procurement of materials, construction and testing of the cryogenic systems for the detector cryostats.

- Split between DOE and non-DOE.

- Responsible parties for non-DOE scope are to be identified.

- LAr procurement of 70 kton. Split between DOE and non-DOE.

- Integration of all components: DOE. CERN integrating detector cavern.

- Participation in the DUNE prototyping effort at CERN (ProtoDUNE-SP and ProtoDUNE-DP). 


\section{Modes of Operations}

- GAr Purge: GAr is slowly flown from the bottom of the tank (initially full of air) to push the impurities out from the top. Reduces contaminants $(\mathrm{O} 2, \mathrm{~N} 2, \mathrm{H} 2 \mathrm{O})$ to ppm level.

- GAr Circulation: GAr is circulated in a closed loop and purified through the GAr purification system. Reduces $\mathrm{O} 2$ and $\mathrm{H} 2 \mathrm{O}$ to sub-ppm level.

- Cool-down: a mix of GAr and LAr is flown into sprayers to generate a mist of small liquid droplets that are circulated by another set of sprayers flowing GAr only.

- Filling: GAr is transferred from surface and re-condensed underground. Once the cryostat and the detector are cold, LAr flows from the condenser into each cryostat.

- Steady state operations:

- LAr is continuously purified via external LAr pumps (4 in each cryostat, all in service to achieve purity, then fewer to maintain purity).

- Boil-off GAr is recondensed in the condensers (outside the cryostat) and purified in the LAr purification system before being reintroduced as liquid.

- Emptying: at the end of operations, the tank is emptied and the LAr removed. 


\section{Selection of Design Parameters}

$$
\text { Lifetime }[s]=\frac{3 \cdot 10^{-13}[s \cdot \text { parts of Oxygen }]}{\text { Contaminant }[\text { parts of Oxygen }]]}
$$

\begin{tabular}{|l|l|}
\hline Design Parameter & Value (per cryostat) \\
\hline GAr Flow rate during piston purge & $254 \mathrm{~m}^{3} / \mathrm{hr}$ \\
\hline Maximum cool down rate detector & $40 \mathrm{~K} / \mathrm{hr}$ \\
\hline Maximum Delta_T any two detector points & $50 \mathrm{~K}$ \\
\hline Maximum available cooling power & $100 \mathrm{~kW}$ \\
\hline Required electron lifetime & $>3 \mathrm{~ms}$ \\
\hline Required LAr purity (Oxygen equivalent contamination) & $<100 \mathrm{ppt}$ \\
\hline Maximum liquid turnover (5 days/volume change) & $36.12 \mathrm{~kg} / \mathrm{s}$ \\
\hline Cryostat operating pressure & $130 \mathrm{mBarg}$ \\
\hline Cryostat design pressure & $350 \mathrm{mBarg}$ \\
\hline
\end{tabular}




\section{Cryostat}

- Membrane cryostat technology.

- $1.2 \mathrm{~mm}$ membrane (304L).

- $0.8 \mathrm{~m}$ of passive insulation (polyurethane).

- Support structure bears $\mathrm{LAr}+\mathrm{GAr}$ loads:

- $12 \mathrm{~mm}$ vapor barrier (stainless steel).

- $1.1 \mathrm{~m}$ high l-beams (steel).

- The design includes the feedback $18.94 \mathrm{~m}$ from the assembly of ProtoDUNE.

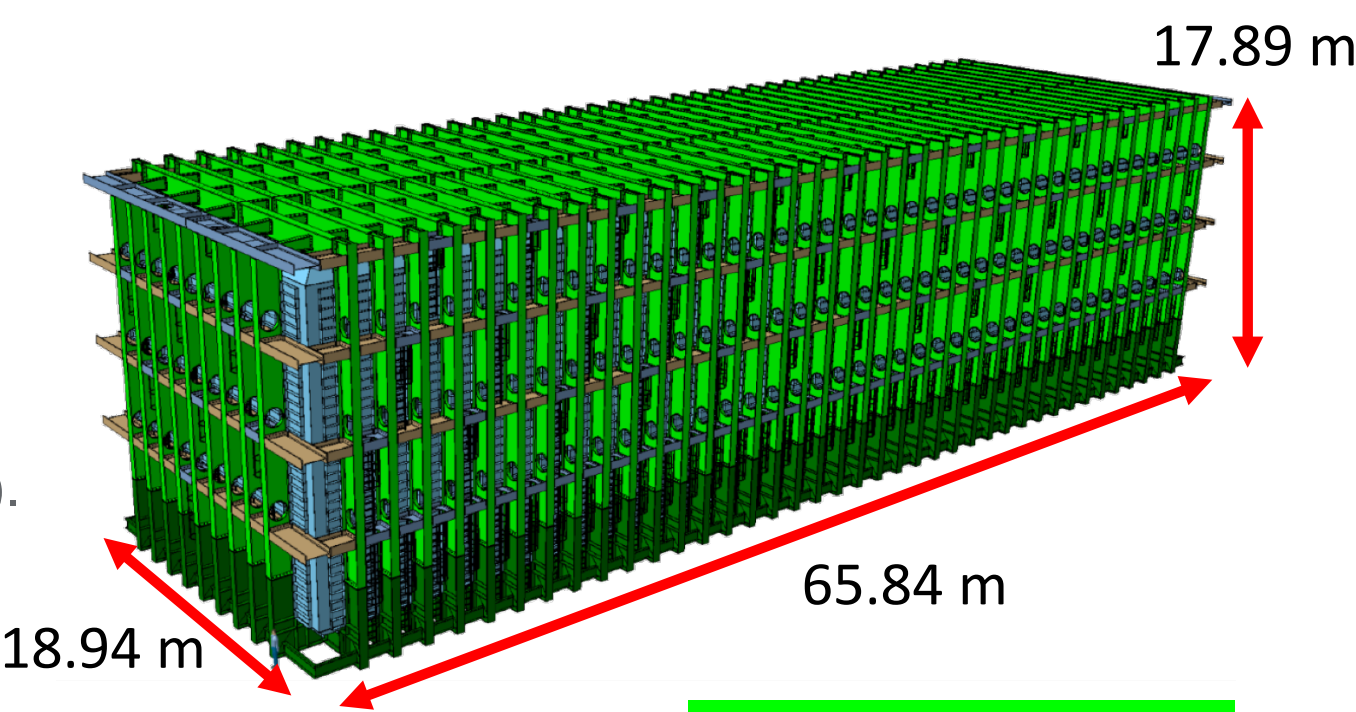

$4 \times 17,100$ tons $\operatorname{LAr}$

\begin{tabular}{|l|c|c|c|}
\hline Dimensions of one cryostat & Length $(\mathrm{m})$ & Width $(\mathrm{m})$ & Height $(\mathrm{m})$ \\
\hline Membrane Internal Dimensions & $\mathbf{6 2 . 0 0}$ & $\mathbf{1 5 . 1 0}$ & $\mathbf{1 4 . 0 0}$ \\
\hline SS plate Internal Dimensions & 63.60 & 16.70 & 15.60 \\
\hline External Dimensions of steel structure & 65.84 & 18.94 & 17.84 \\
\hline
\end{tabular}




\section{Cryostat feedthroughs (DRAFT)}

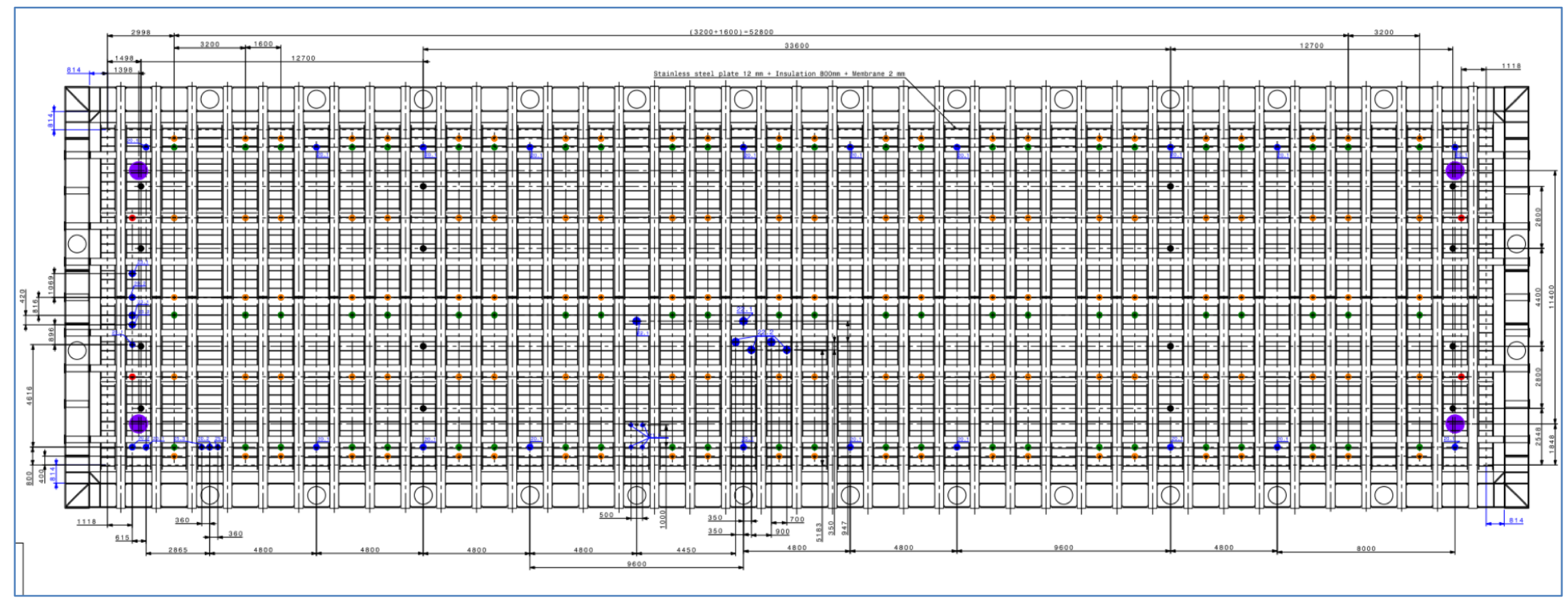




\section{Cryogenics Systems}

- Infrastructure Cryogenics (INF):

- Receive Ar/N2.

- Transport Ar to cavern.

- LN2 refrigeration (compressors, cold boxes, N2 distribution system).

DOE

- Proximity Cryogenics (PROX) :

- Circulate and purify LAr.

- Achieve and maintain LAr purity.

- Recondense and purify boil off GAr.

Non-DOE

- Internal Cryogenics (INT):

- Inside the cryostat.

- Cryostat purge, cool down, fill.

- GAr/LAr distribution. 
Cryogenics Process Flow Diagram

NO cryogens in the shaft.

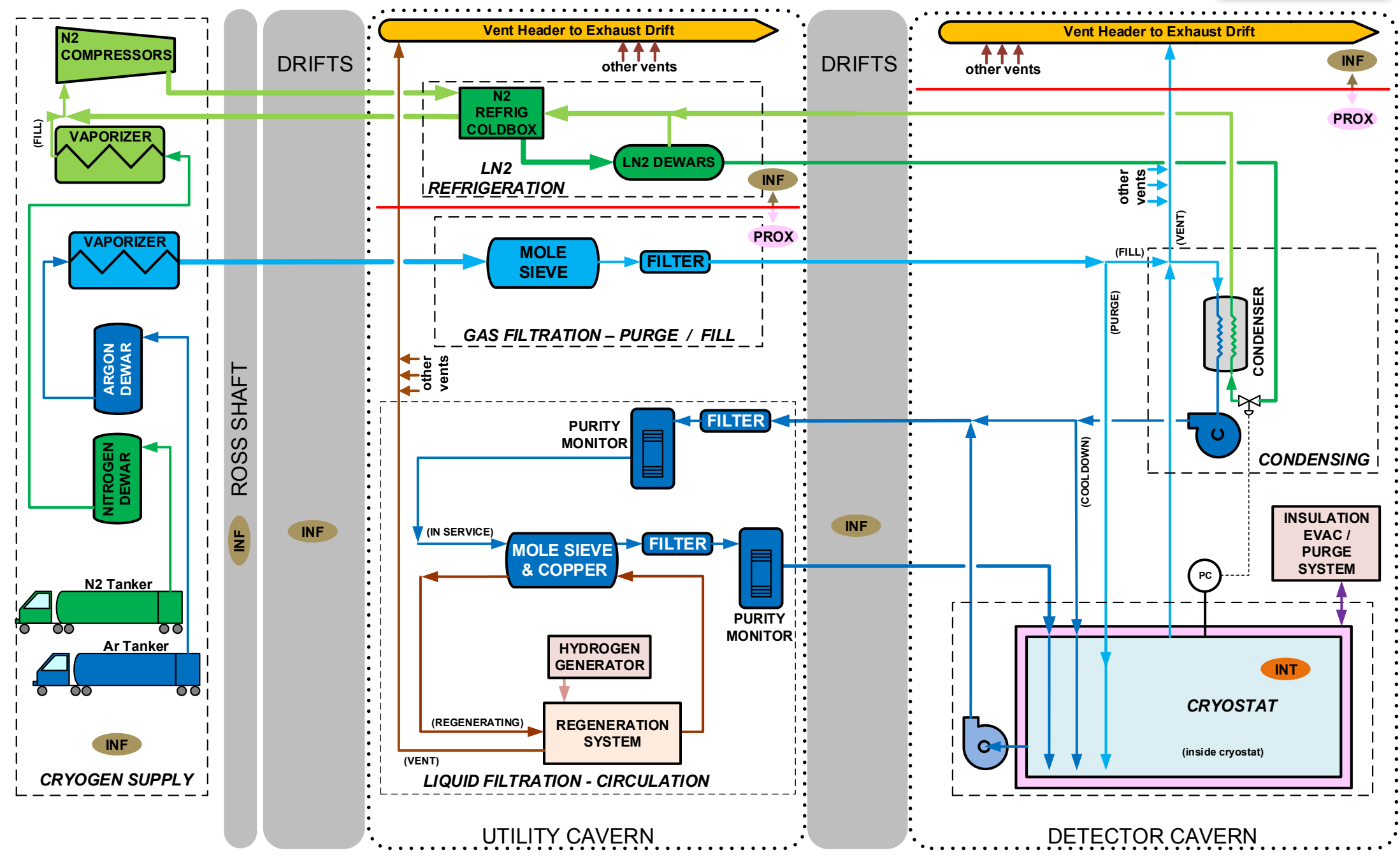




\section{Infrastructure Cryogenics}




\section{Infrastructure Cryogenics - Receiving Facilities}

Current Cryogens Storage and vaporizers: $2 \times 50 \mathrm{~m}^{3}$

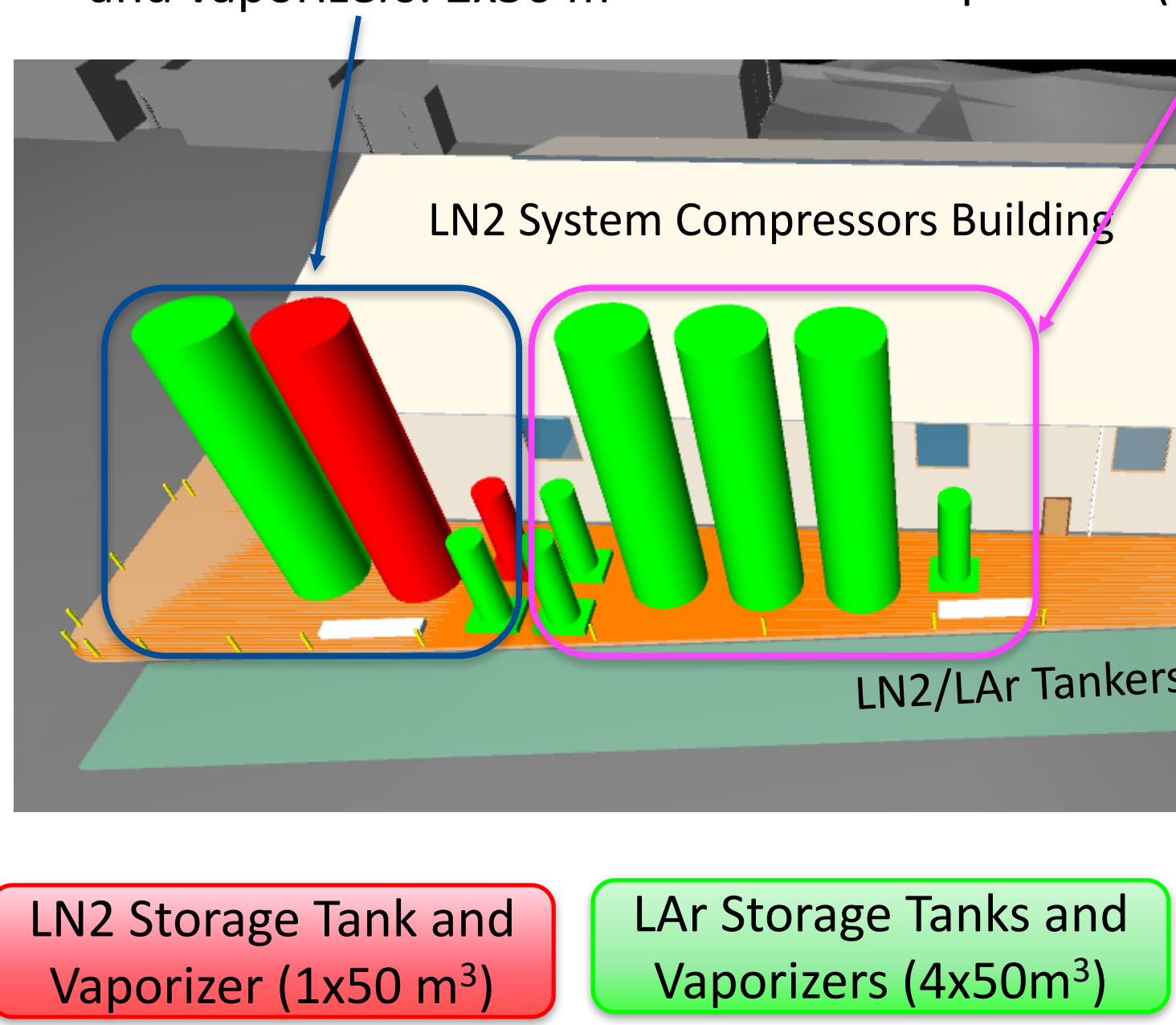

Proposed additional temporary LAr Storage and Vaporizers (LAr fill only): $3 \times 50 \mathrm{~m}^{3}$ 


\section{Infrastructure Cryogenics - LN2 Refrigeration System}

- 4 Commercial units:

- Cold boxes and gas boosters in the cavern.

- GN2 compressors above ground.

- Units assembled in cavern, based on transport limits.

- 3 units for cryostats 1, 2 and $4^{\text {th }}$ unit added for cryostats 3,4 .

- $4 \times 97 \mathrm{~kW}$ units.

- Refrigeration model validated with COCOCOFE simulator.

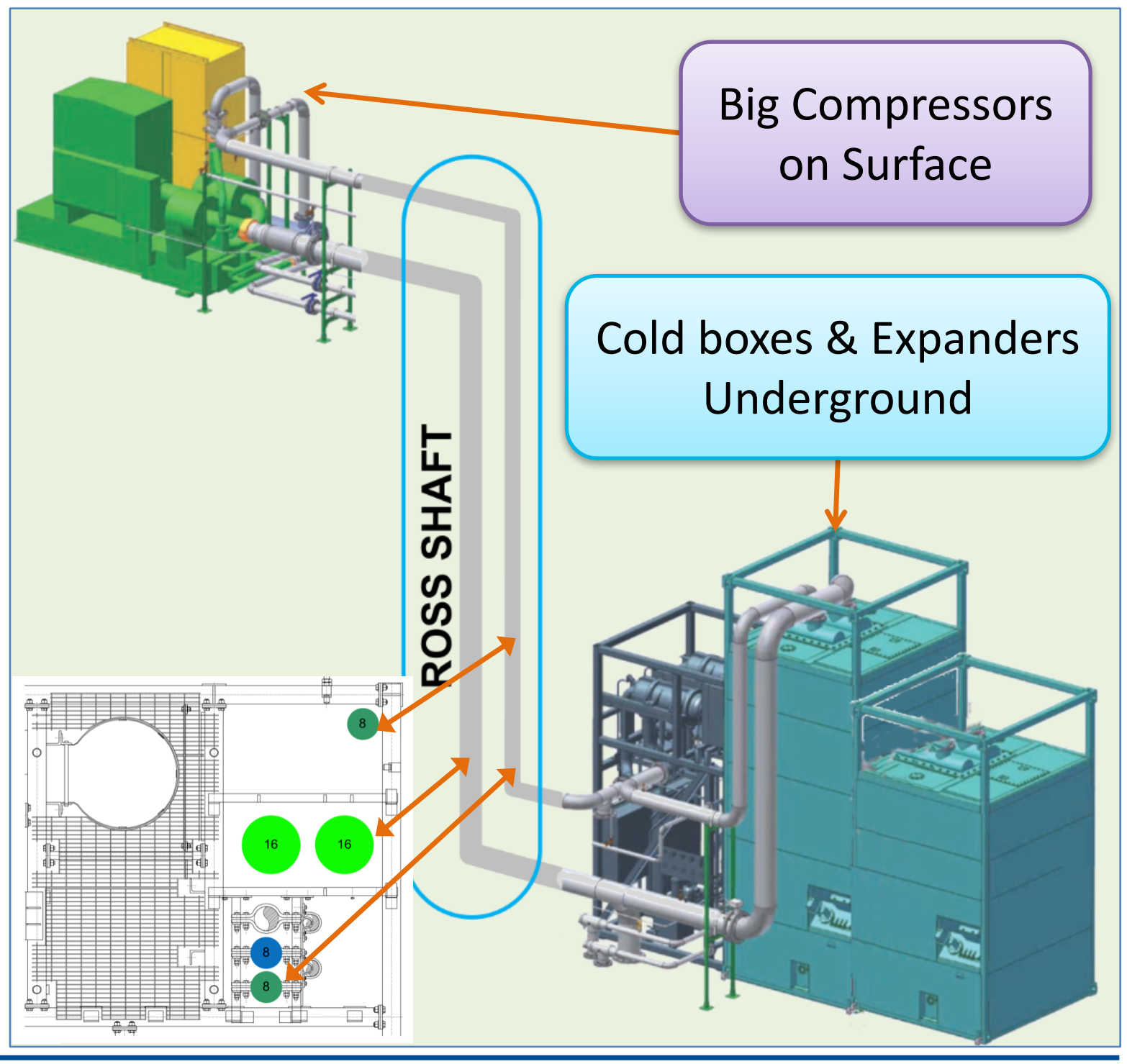




\section{Infrastructure Cryogenics in Central Utility Cavern (CUC) - LN2 Refrigeration and Storage}

- Lifting eyes in the ceiling of CUC for LN2 cold boxes and booster compressors positioning and maintenance.

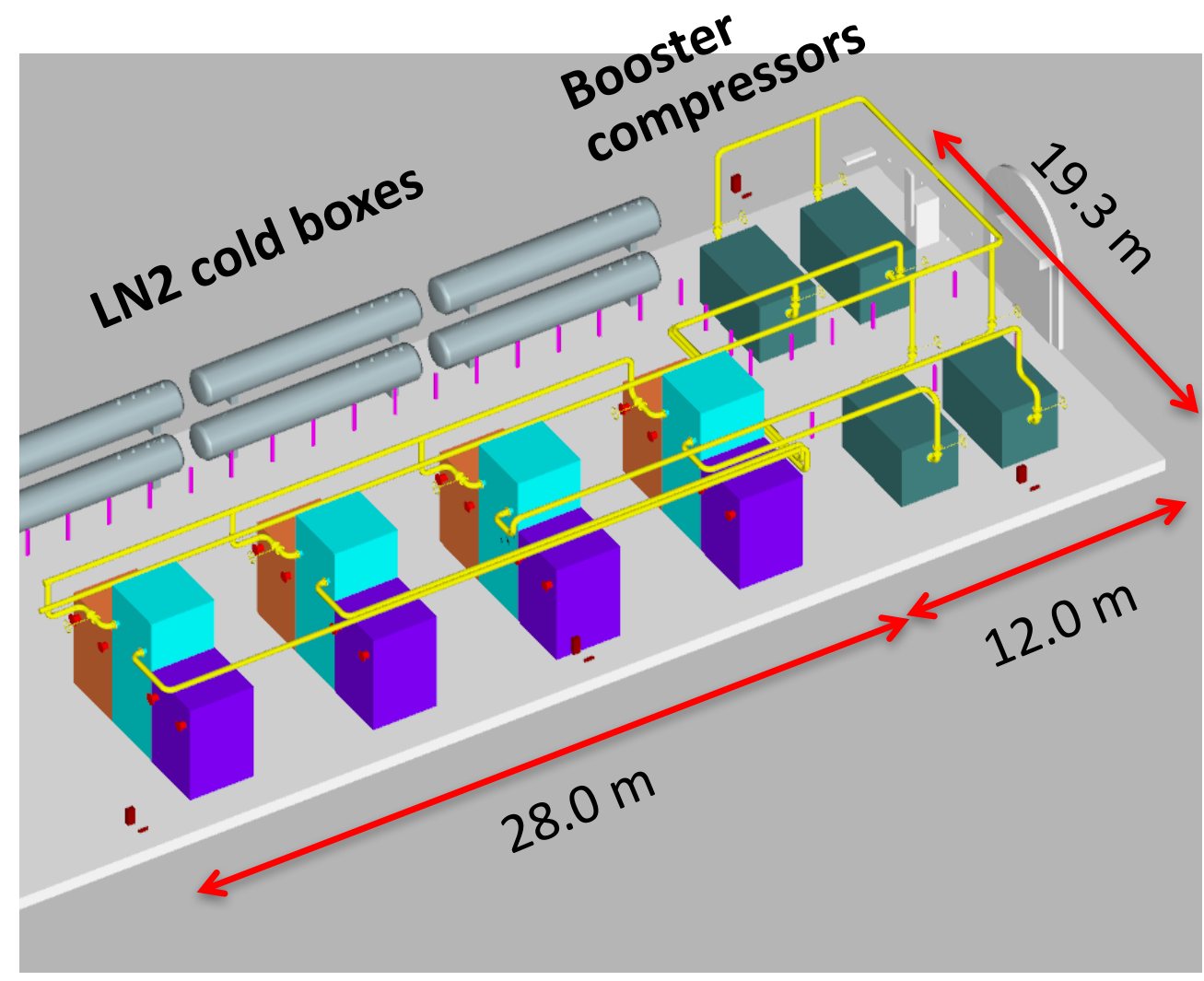




\section{LN2 Refrigeration System - Procurement Strategy}

- Chosen design/fabricate/install phased approach:

- Base $\rightarrow$ Design (full scope: 4x97 kW units) and Fabrication of first three units only (required for Detectors \#1, \#2).

- Option $1 \rightarrow$ Installation and Commissioning of first three units.

- Option $2 \rightarrow$ Fabrication of 4th unit (required for Detectors \#3, \#4).

- Option $3 \rightarrow$ Installation and Commissioning of 4th unit.

- Iterating with DOE on Acquisition Plan (AP) for the whole LN2 system.

- Writing Functional Requirements Specifications (FRS).

- Goal is to issue the Request For Proposals (RFP) to industry by Dec 2017 and award the contract by Jun 2018 (subject to availability of funds). 


\section{Proximity Cryogenics}




\section{Proximity Cryogenics in Detector's Cavern - Mezzanine}

- Cryostat Pressure Safety Valves (PSVs), piping and lockout valves.

- Small LAr buffer tanks (for condenser pumps) and condenser LAr pumps.

- LAr phase separators (return from purification).

- LN2 phase separators and condensers.

- Frame for warm panels (being filled with valves, etc.).

- Interconnecting piping.

- PLC racks in situ.

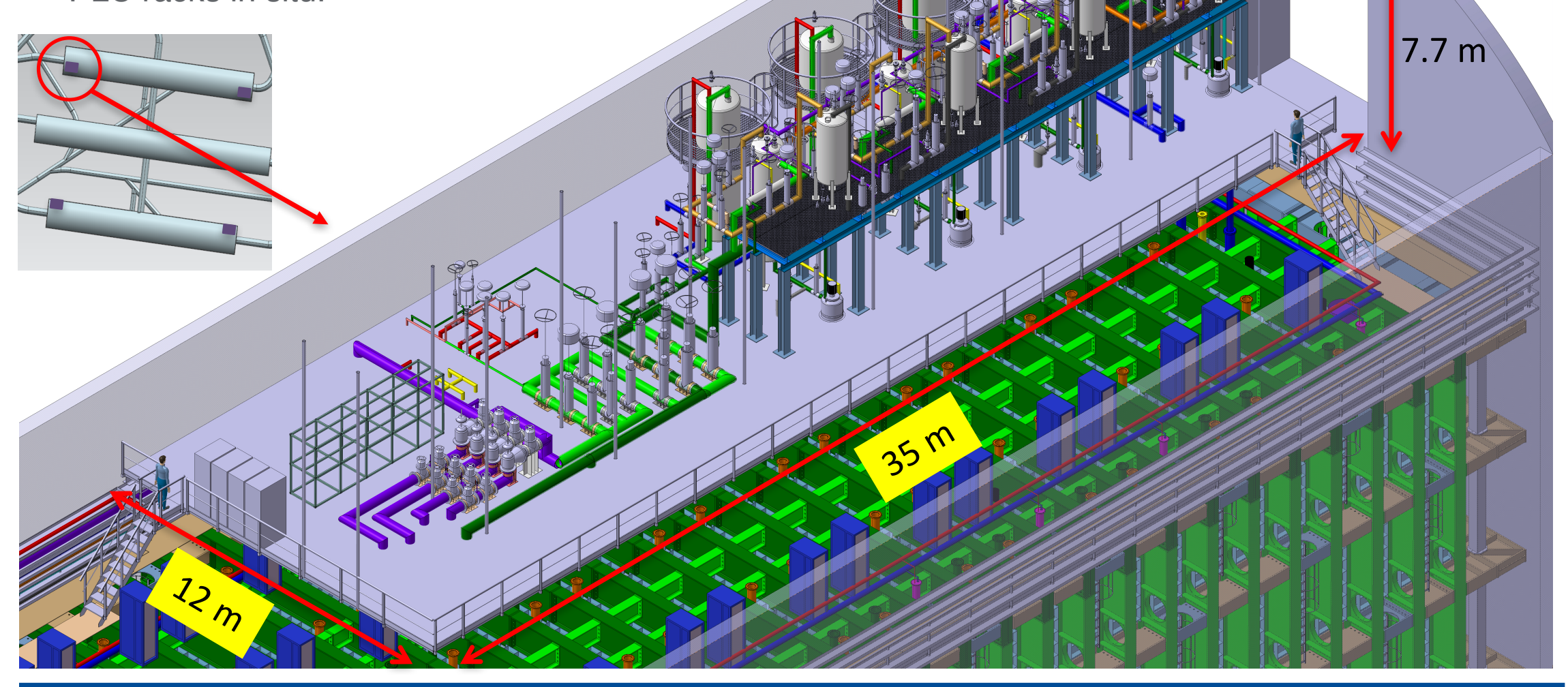




\section{Proximity Cryogenics in Detector's Cavern - LAr Pumps}

- LAr pumps.

- Concept of Clean Room (CR) for detector installation.

- Inline safety valves (one per penetration). Same as in ProtoDUNEs.

Cryostat Secondary Barrier
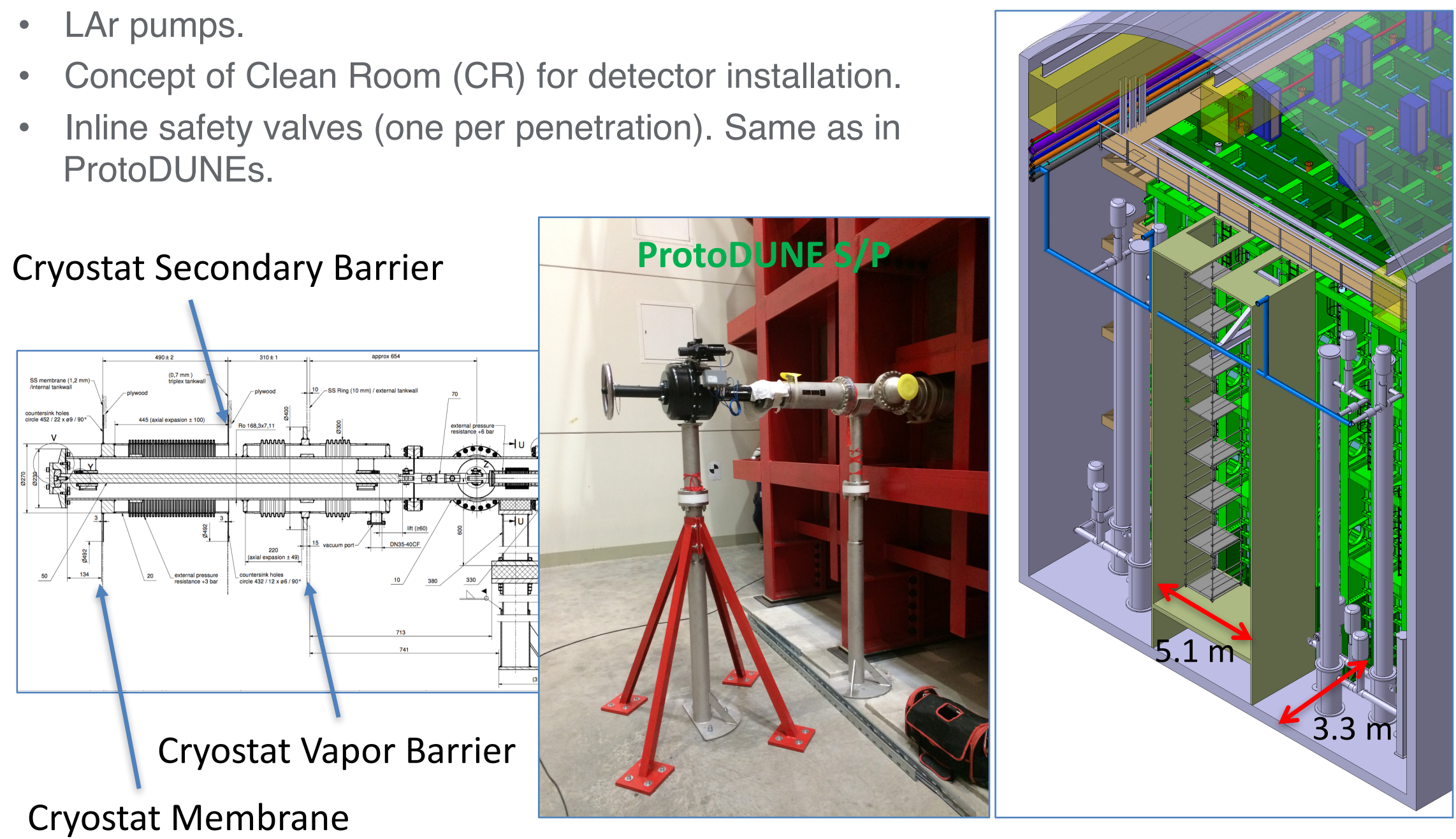


\section{Proximity Cryogenics in the CUC - Layout}

\section{Detectors \#3, 4}

\section{Detectors \#1, 2}

\section{LN2 Refrigeration System}

\section{LAr Purification 3 LAr Purification 4 LAr Purification 1 LAr Purification 2 LN2 Storage Dewars}
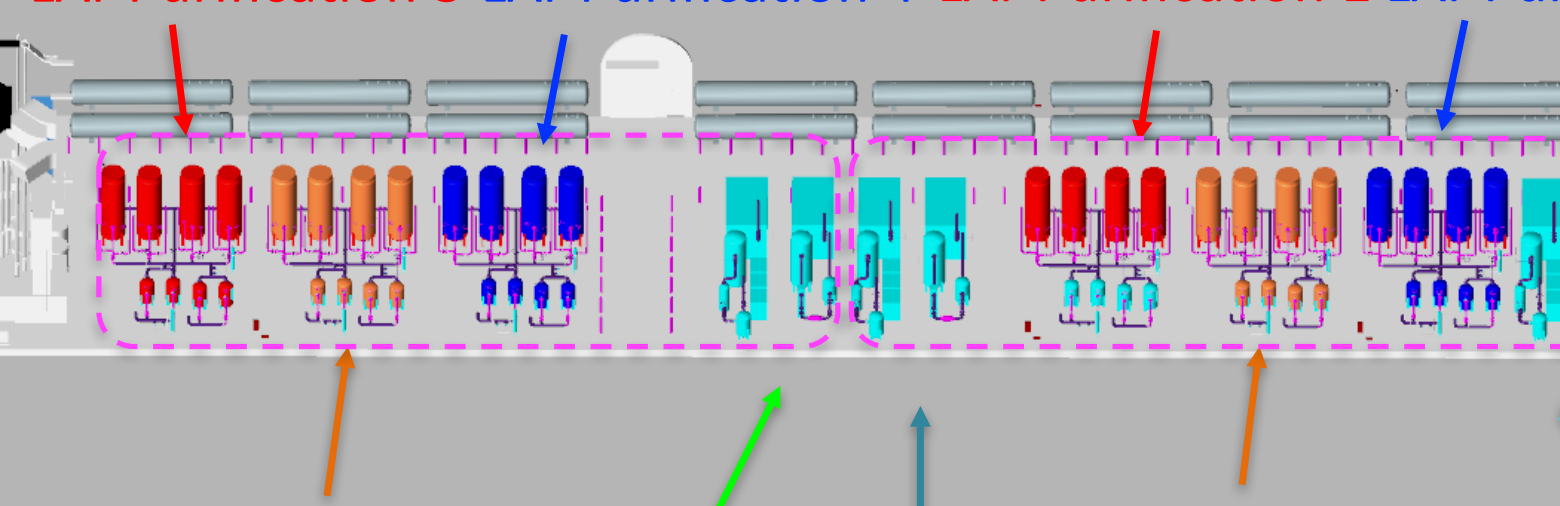

GAr Purification 3, 4
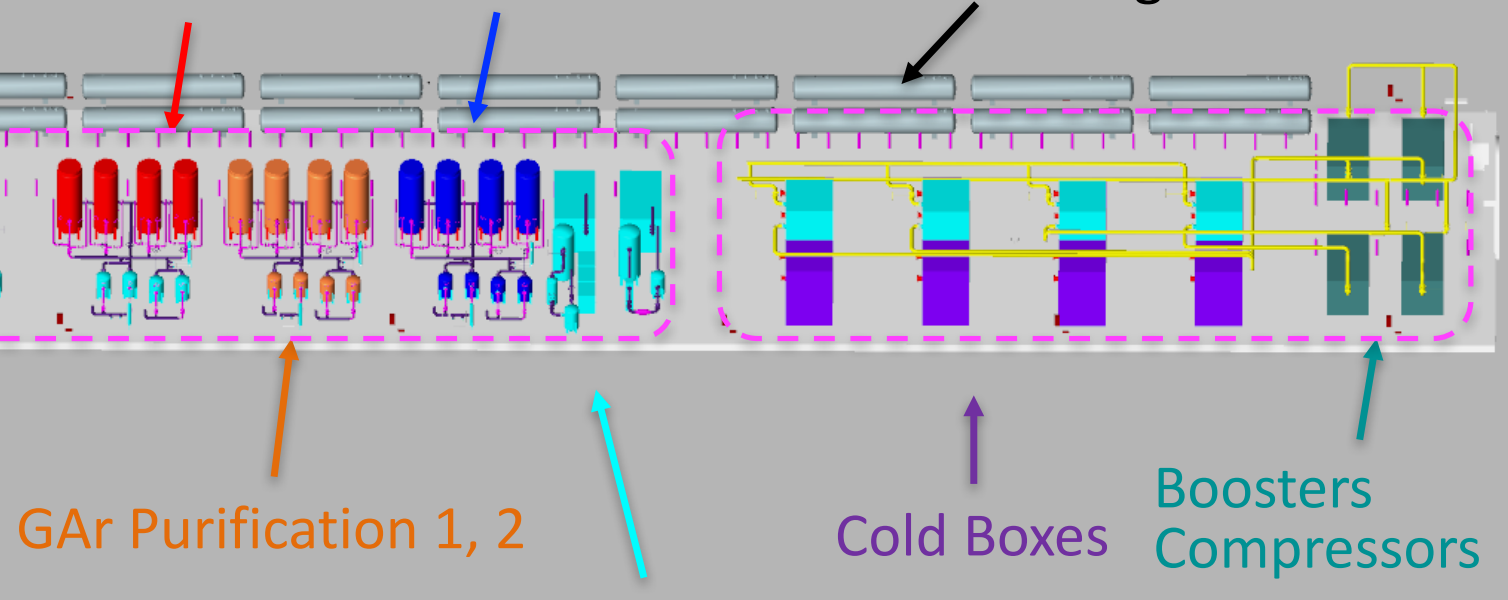

Regeneration 3, 4 Regeneration 1 


\section{LAr Procurement}

- Contracted with a consultant to develop LAr procurement strategy and refine cost and schedule estimates.

- Required Purity: less than 10 ppm Water, 5 ppm Oxygen, 10 ppm Nitrogen (1 ppm would be desirable for N2, but not needed as a requirement).

- Assuming can max out the LN2 refrigeration capacity.

- In Dec 2016 we have visited the five major US LAr suppliers.

- Key takeaway:

- Supply is feasible, even likely coming from several hundreds miles away.

- New plants capable of producing LAr put in service since previous analysis when it was likely coming from 1,000 miles away (reduced travel distance).

- Currently no single supplier, probably 2-3 suppliers either contracted separately or as subs of a LAr procurement coordinator.

- Gathered very useful information for the development of the LAr receiving facility, especially the amount of storage.

- Reviewing replies to a Request For Information (RFI) issued to collect more information about how to structure this procurement, current costs and how to project them in the future. 


\section{Cryogenics Schedule Summary Overview}

Dec-19

Feb-28

CD-2/3b Project

CD-4 (early

Baseline \& FS Constr. Approval

completion)

Cryogenic\$ Prelim Design

Cryogenics Final Design

Start of cryo installation

\$.0. Chamber \#1/ Start Install Cryostat \#1

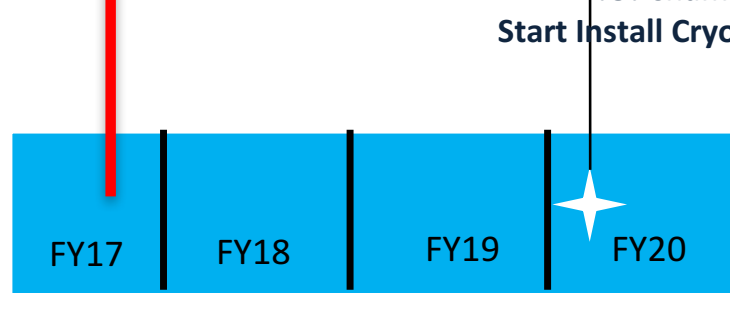

DOE Activity

DOE and Non-DOE Activity

Non-DOE Activity

Fabricate and Deliver Cryogenic Equipment

Install Cryo Equipment - Surface Facilities

Install LN2 Plants \#1-3

VIIIIIS GAr Filtration Install

LN2 System Piping/Valves Install

VIIIII Cryogenic Equipment for Cryostat \#1

VIIIIIA Cryogenic Equipment for Cryostat \#2

VIIIIA Cryogenic Equipment for Cryostat \#3

VIIIIIIIS Cryogenic Equipment for Cryostat \#4

Purge, Cooldown \& Fill Cryostat \#1

Purge, Cooldown \& Fill Cryostat \#2

Purge, Cooldown \& Fill Cryostat \#3 UIIIIIIIII

Purge, Cooldown \& Fill Cryostat \#4

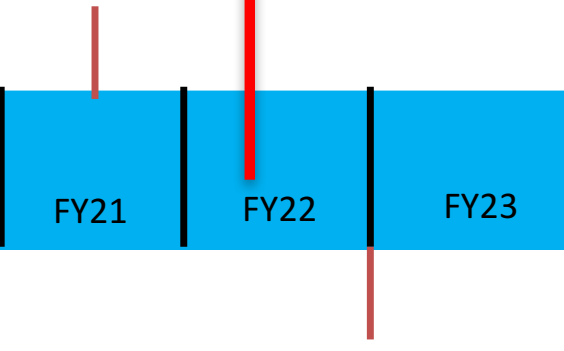

Cryostat \#1 Ready for Detector Installation

Det \#1 Commissioned

Det \#2 Commissioned 


\section{Summary and Next Steps}

- Advancing the design of all parts focusing on requirements and interfaces, in particular between Cryostat, Cryogenics and Conventional Facilities. Expected sign-off on cryostat feedthroughs early Nov 2017 to being preliminary study with GTT early 2018 .

- Identified the equipment layout and the spaces needed in each zone (above ground, shaft, detector cavern, central utility cavern, drifts).

- Studied the logistics from above ground to underground and we can deliver all the components to their location.

- Working on a design/fabricate/install strategy for the LN2 system. Iterating with DOE and writing Functional Requirements Specifications. Goal is to award a phase funded contract by Jun 2018.

- Contracted with a consultant to develop a strategy for LAr procurement. Reviewing replies from the industry to RFI. Process also informing the development of the receiving facilities on the surface.

- Developing the Proximity Cryogenics on the mezzanine to inform the cryostat design.

- In parallel, continuing the prototyping effort to inform the LBNF/DUNE design, fabrication, installation.

- Ground breaking in Jul 2017! Planning to start cryo installation at SURF in 2022. 


\section{Thanks}
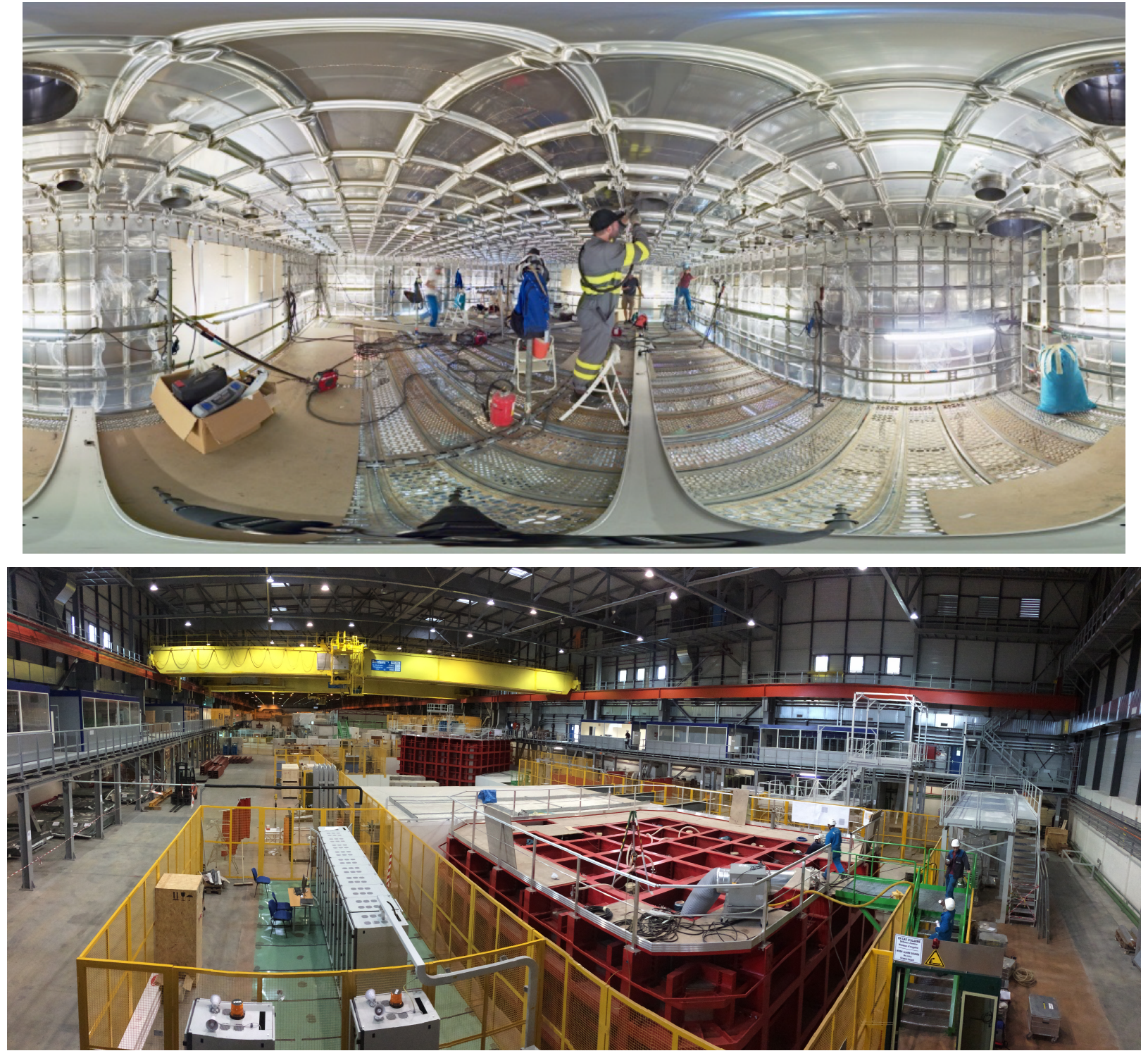


\section{Backup Slides}




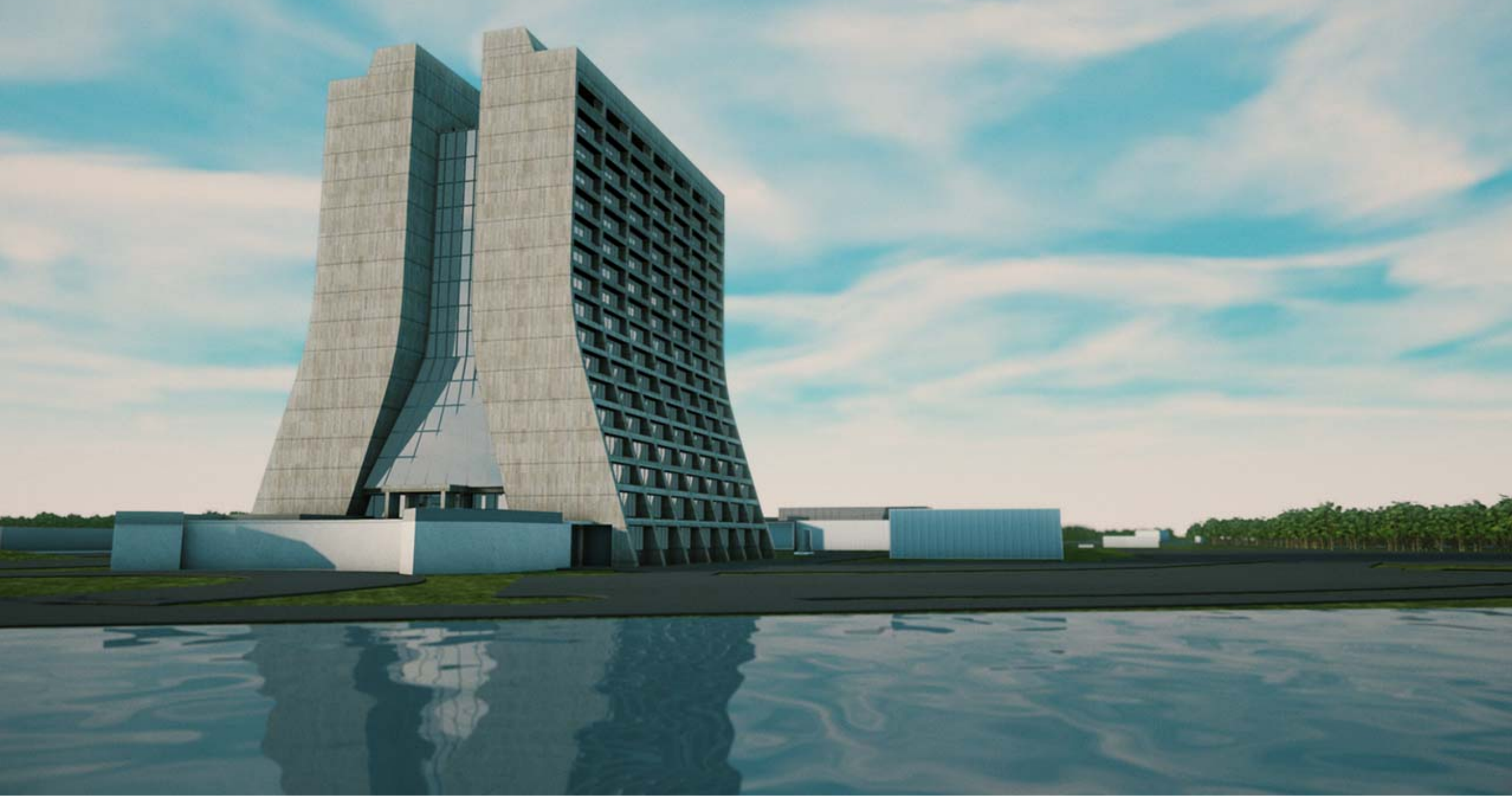

$32 \quad 09.13 .17$ 


\section{Cooling Power Requirements under different scenarios}

\section{$4 \times 97 \mathrm{~kW}$ units $\rightarrow$ Only 3 needed in purity maintenance mode, one full spare unit.}

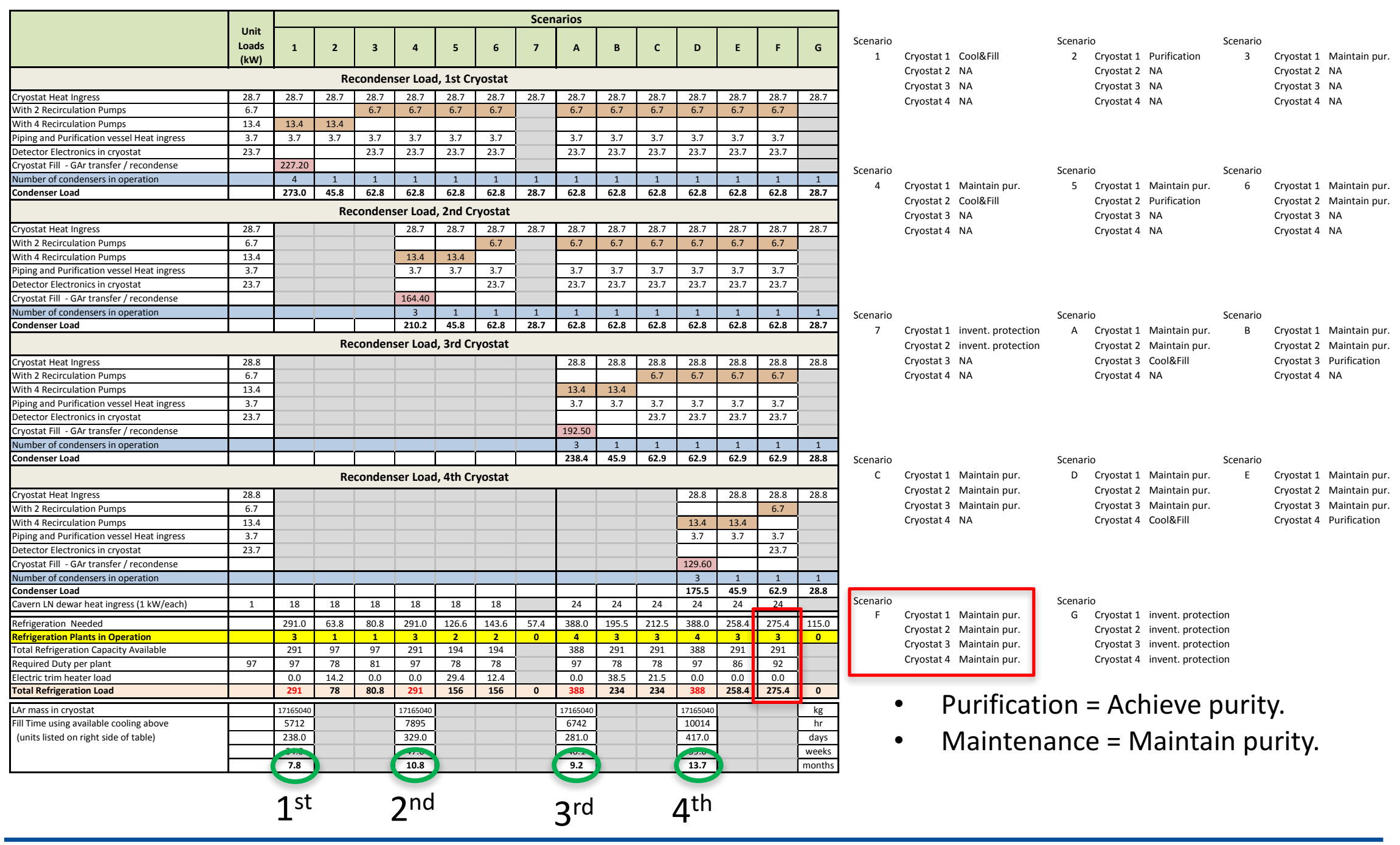




\section{Internal Piping}

- We have modeled the feedthroughs through the roof of the cryostat and are now modeling the internal piping.

- At the bottom of the cryostat there will be:

- LAr return lines (across the length of the cryostat).

- GAr purge lines (across the length or the width of the cryostat).

- At the top of the cryostat there will be:

- Cool down penetrations (currently 10 each side, but it could change). Each one contains:

- LAr/GAr pipes with nozzles at the end.

- GAr momentum pipes with nozzles at the end.

- GAr boil-off.

- GAr line to PSVs.

- GAr Make-up.

- LAr emergency return. 


\section{Side View}

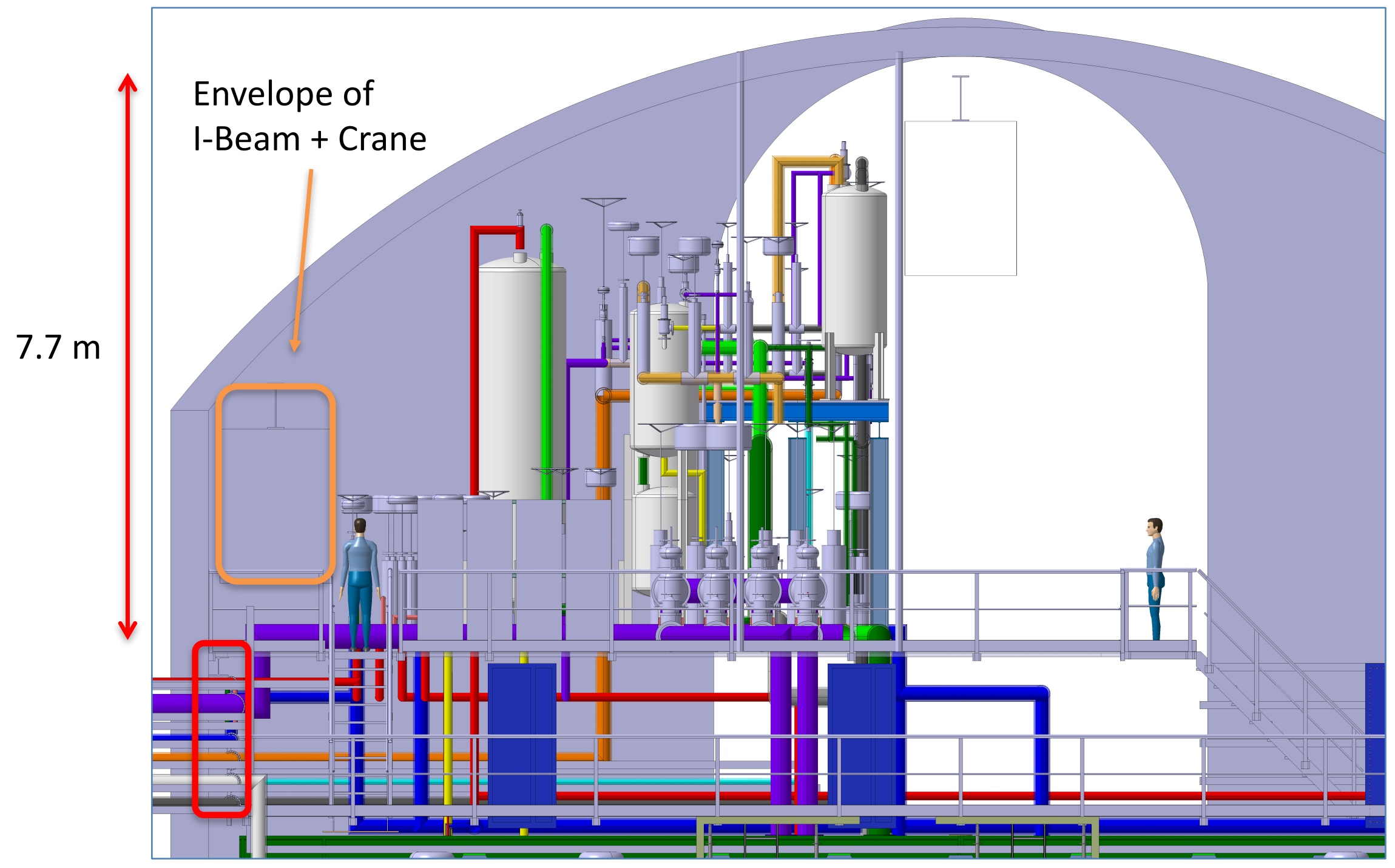




\section{Top View}

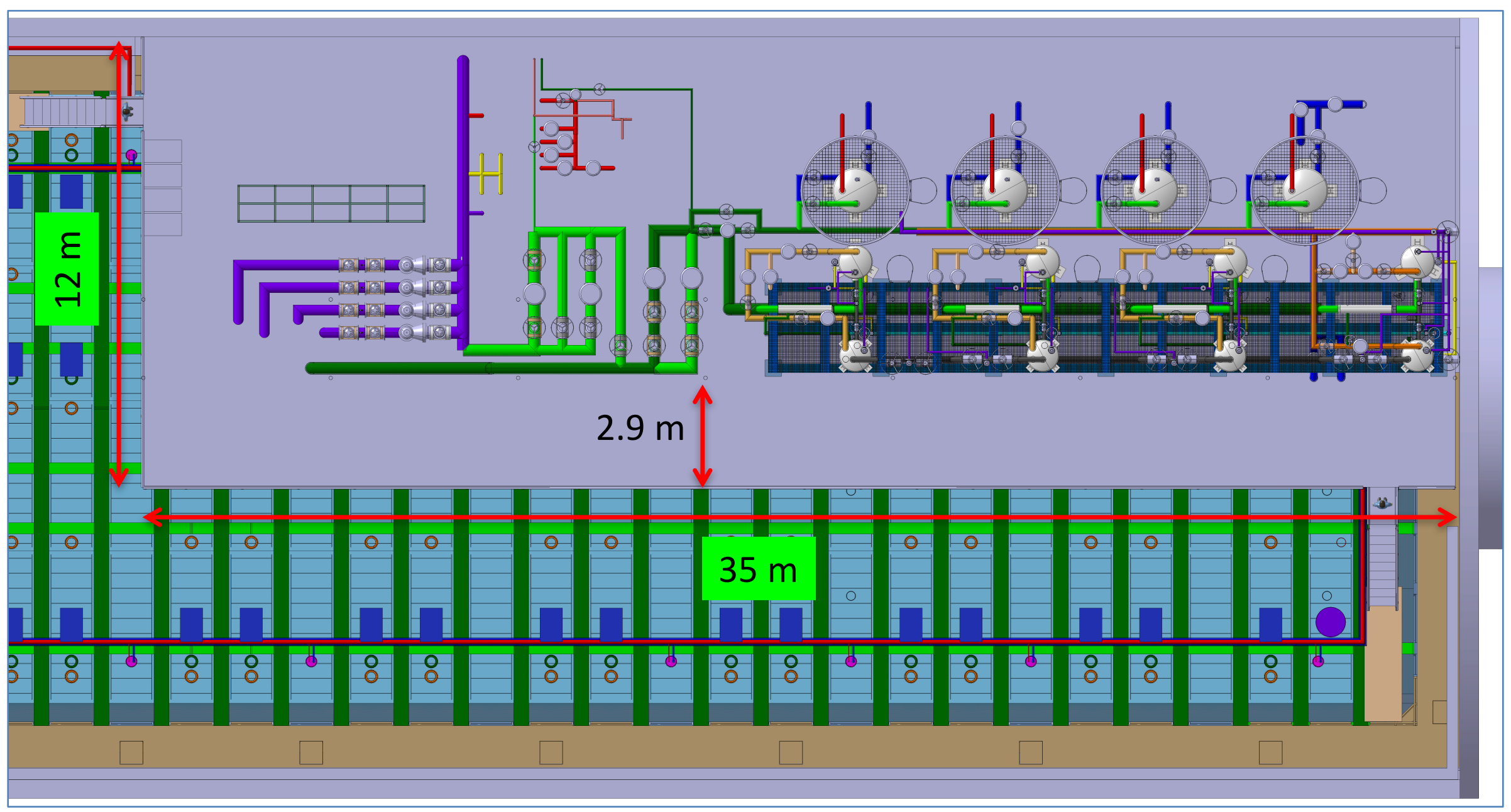




\section{Infrastructure Cryogenics - Piping in the Shaft}

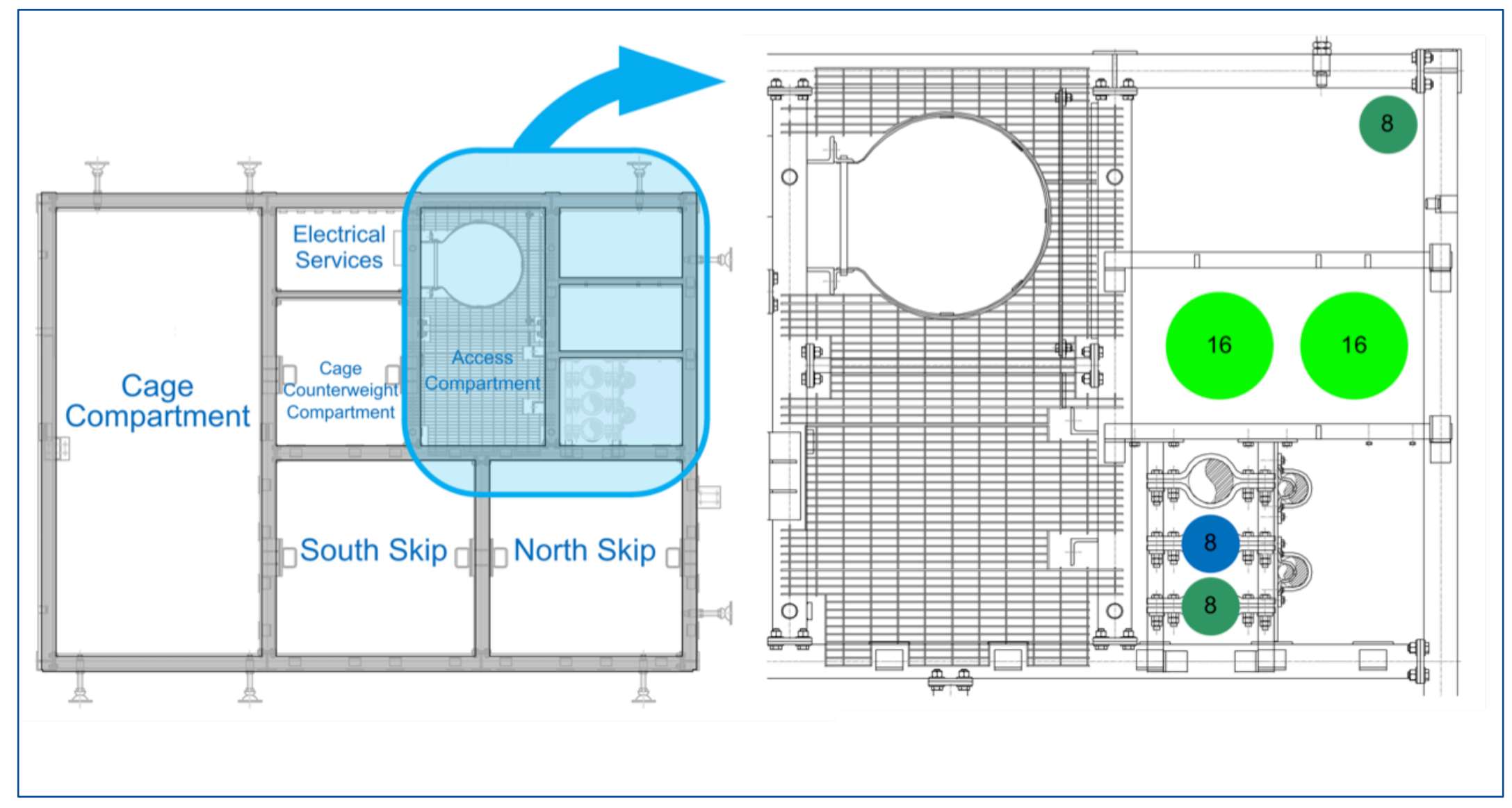




\section{Infrastructure Cryogenics on Surface - Nitrogen Compressors}

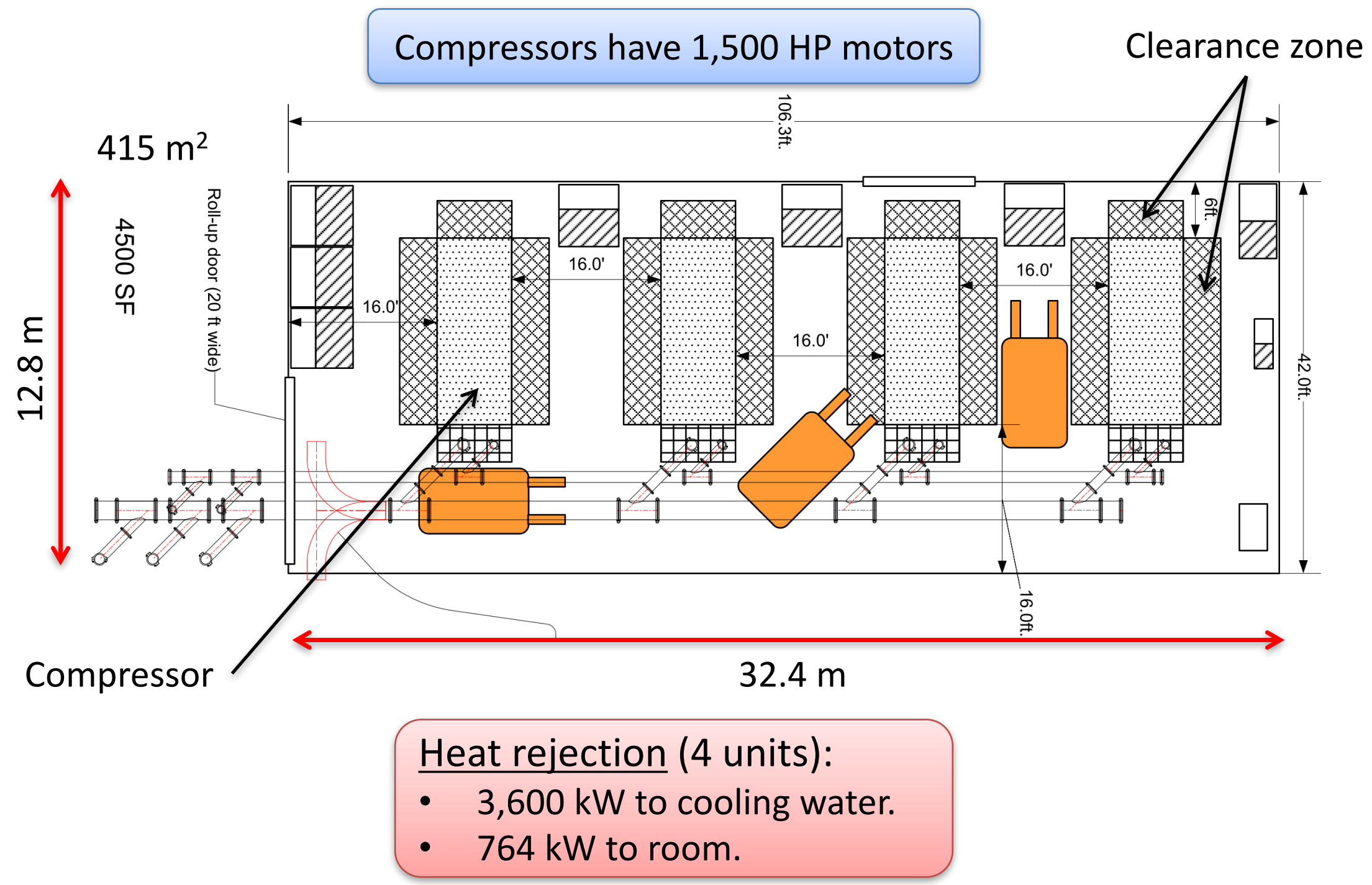




\section{Infrastructure Cryogenics in Central Utility Cavern - Cold boxes}

- Commercial cold boxes.

- Assembled in cavern.

- Assembly sections based on transport limits.

- 3 units for cryostat 1,2 and $4^{\text {th }}$ unit added for cryostat 3,4 .
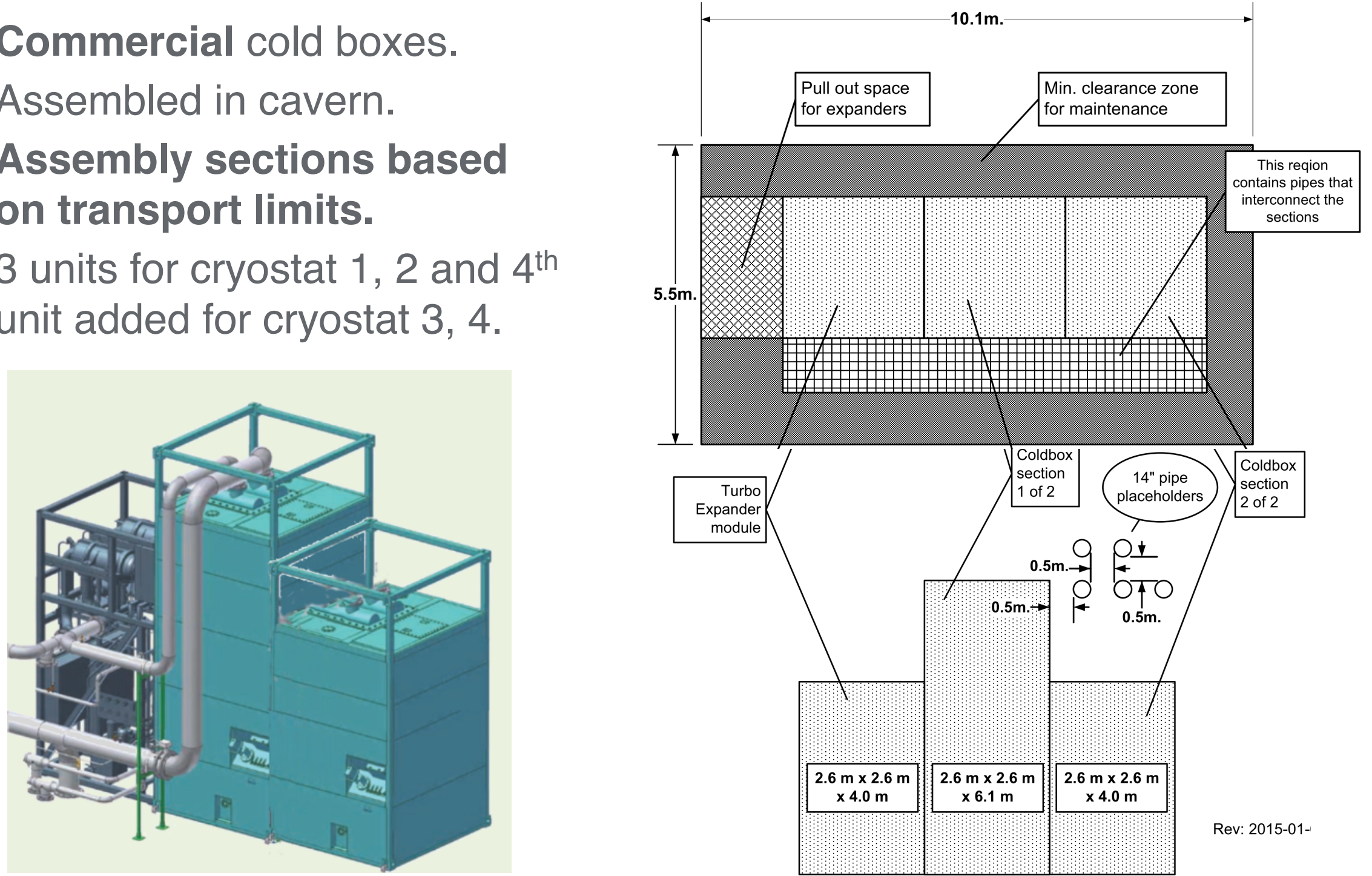


\section{Infrastructure Cryogenics}

- Main items:

- Cryogens receiving station (surface).

- GN2/GAr piping in the shaft.

- LN2 refrigeration system.

- LN2 storage dewars (underground).

- Interconnecting piping, buffer tanks, valves, instrumentation, etc. 


\section{Proximity Cryogenics}

- Main items:

- LAr circulation pumps (Detector cavern).

- Condensers for boil-off GAr (Detector cavern).

- LN2/LAr Phase Separators (Detector Cavern).

- GAr/LAr Purification System with regeneration (CUC).

- Interconnecting piping, buffer tanks, valves, instrumentation, etc. 


\section{Central Utility Cavern}

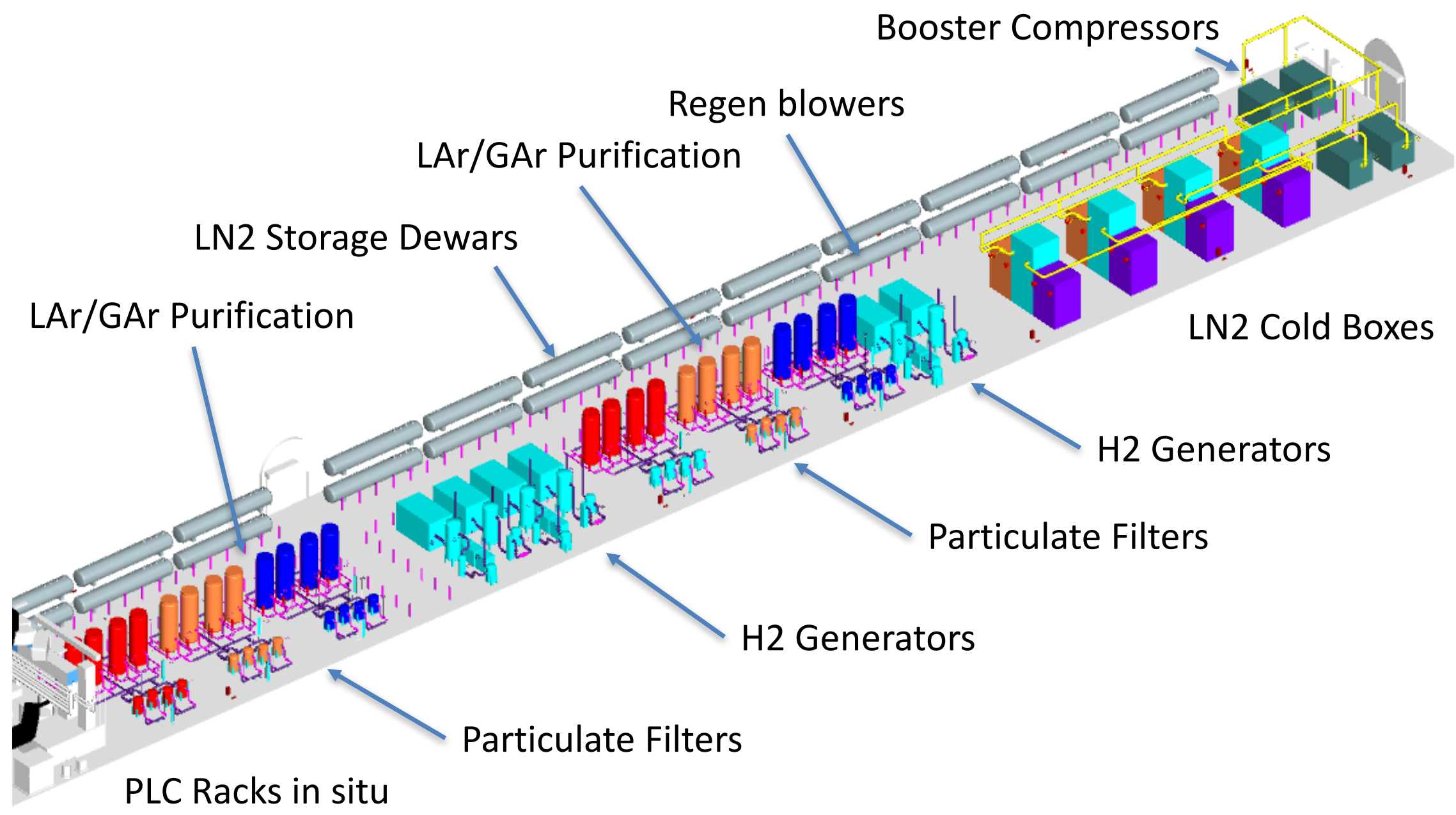




\section{Liquid filtration}

- Mole Sieve

- Water capture

- Copper

- Oxygen capture

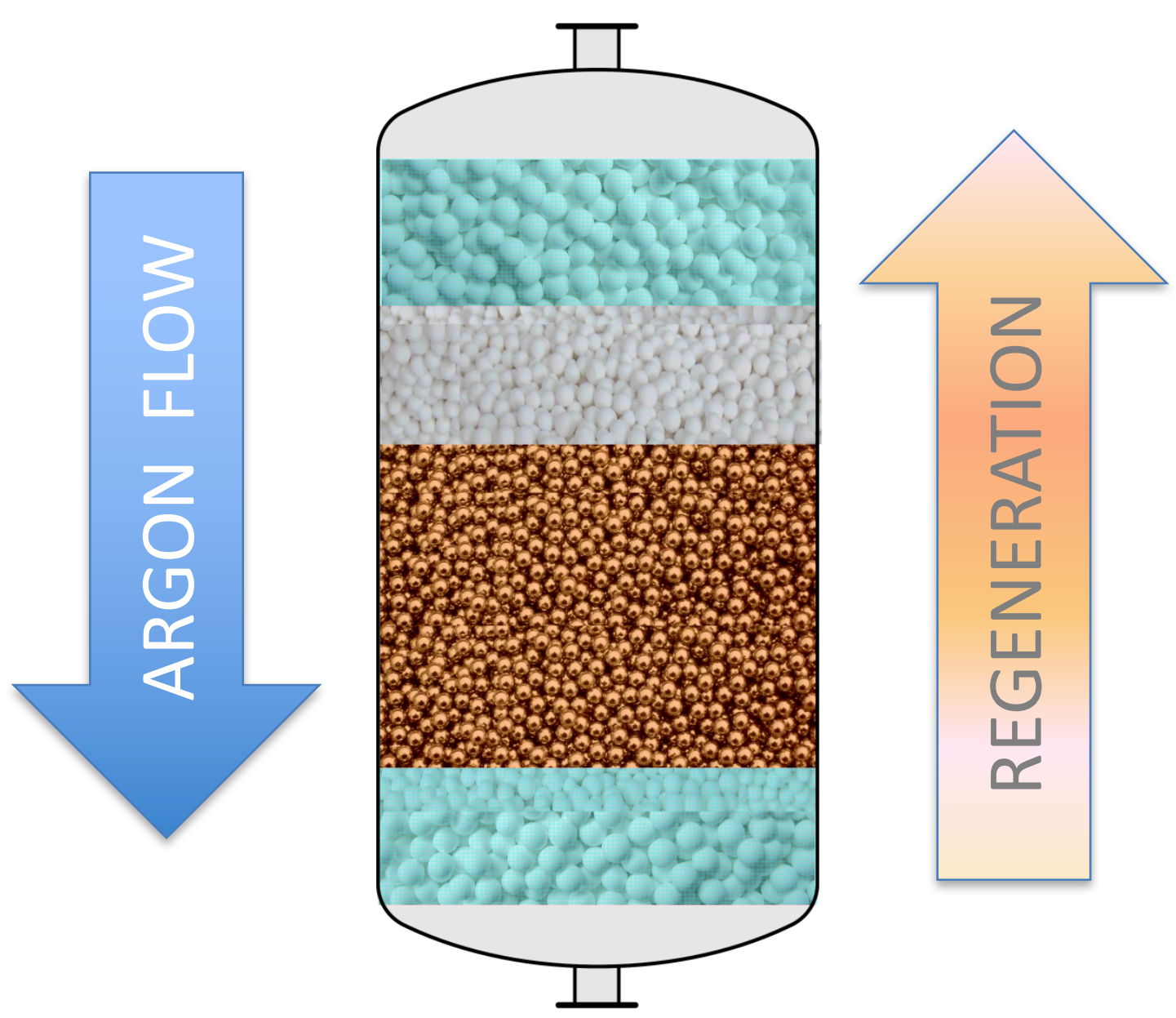




\section{Strategy}

- We exploit the large expertise existing at CERN and Fermilab (PAB, LAPD, 35 ton, MicroBooNE, ATLAS, WA105 1x1x3), but also in the LN2/LNG industry.

- We use commercial items to the extent possible (e.g. LN2 refrigeration, LAr circulation pumps).

- We have built our own process simulation models using COCOCOFE, for mass and energy balance. 